Volume 8

Issue 3 Humanitarian Technologies and

Genocide Prevention

Article 5

October 2014

\title{
No More Hidden Secrets: Human Rights Violations and Remote Sensing
}

Tommy O'Connell

Salem State University, Department of Geography

Stephen Young

Salem State University, Department of Geography

Follow this and additional works at: https://digitalcommons.usf.edu/gsp

\section{Recommended Citation}

O'Connell, Tommy and Young, Stephen (2014) "No More Hidden Secrets: Human Rights Violations and Remote Sensing," Genocide Studies and Prevention: An International Journal: Vol. 8: Iss. 3: 5-31.

DOI:

http://dx.doi.org/10.5038/1911-9933.8.3.3

Available at: https://digitalcommons.usf.edu/gsp/vol8/iss3/5

This Articles is brought to you for free and open access by the Open Access Journals at Digital Commons @ University of South Florida. It has been accepted for inclusion in Genocide Studies and Prevention: An International Journal by an authorized editor of Digital Commons @ University of South Florida. For more information, please contact digitalcommons@usf.edu. 
No More Hidden Secrets: Human Rights Violations and Remote Sensing

Acknowledgements

The authors would like to thank the DigitalGlobe Foundation for providing a generous satellite imagery grant, for which this study could not have been completed. 


\title{
No More Hidden Secrets: Human Rights Violation and Remote Sensing
}

\author{
Tommy 0'Connell \\ Salem State University, Department of Geography \\ Salem, MA, USA \\ Stephen Young \\ Salem State University, Department of Geography \\ Salem, MA, USA
}

\begin{abstract}
:
Aim: This study used both high resolution and medium resolution satellite imagery to test three semi-automated remote sensing methods, in an attempt to identify useful tools to support eye-witness testimony and reports on human rights violations. As huts are routinely burned down during attacks on a village, particularly in Sudan, the number of huts and villages burned can be used to corroborate on-the-ground reports.

Methods: Three remote sensing methods (Supervised Classification, Change Detection, and Feature Extraction) were performed on imagery from both before the attacks in February 2006 and after the attacks to examine any useful trends that could be used by researchers when manually examining the satellite data.

Results: In general, Supervised Classification methods performed unsatisfactorily. Some classes, such as water and agriculture, had high accuracy rates; however pixels classified as villages performed poorly. The Change Detection method delivered inconclusive results. Feature Extraction, when combined with the kernel density "hot spot" method, appears to be a useful tool to identify villages. The after imagery had no surviving villages in the study area, so Feature Extraction could not be completed on the after imagery and an accurate assessment of the method cannot be fully ascertained.

Conclusions: The results of this study indicate that Feature Extraction is a useful ancillary tool to the practice of manual identification, particularly in places similar to Darfur with semi-arid grassland and rural huts and villages. In addition, the kernel density function when paired with Feature Extracted is a useful tool for visual analysis of clustered villages.
\end{abstract}

Keywords: human rights, genocide, remote sensing, GIS, satellite imagery, Darfur, Sudan

\section{Introduction}

Mass violence and human rights violations have been occurring throughout human history, but only recently has the prospect come forward of using advanced technology to study, prosecute, and possibly prevent these crimes from happening. There is continued debate about the knowledge that Western countries had about the Holocaust, ${ }^{1}$ but it can be argued that the genocide committed by the Nazis during World War II (the murders of Jews, Soviets, Romani, homosexuals and Jehovah's Witnesses, among others) continued to be a "terrible secret" because the international community lacked adequate and compelling information to act. ${ }^{2}$ Some articles bring up the situation of the 1994 mass killings in Rwanda as evidence that information alone may not be sufficient enough to move the international community to act. The beginnings of the Rwandan genocide were reported in the Western press, and when the killings began, a small United Nation (UN) force reported from their position in Rwanda, and CNN and other media sources broadcast continually about the problems. UN Secretary General Boutros Boutros-Ghali appealed for the international community to stop the killings, but there was no large-scale action taken by any nation or the UN. ${ }^{3}$

However, many scientists have been trying to use technology to push public action. There are numerous studies on using satellite imagery and remote sensing to study mass violence and its direct and indirect effects, like refugee camps, burnt villages, or the disappearance of agriculture. As satellite technology grows and develops, the idea of creating an early warning system becomes more of a possibility. Projects like Amnesty International's Eyes on Darfur and Google/US Holocaust Museum's Crisis in Darfur both showcased satellite imagery and analysis to the general public to help persuade and educate. In a one year timeframe from $2007-$ 2008, over 1.2 million individuals visited Amnesty International's website to learn about the Darfur crisis. Amnesty International identified twelve at-risk villages that were vulnerable to violence. The website has imagery of these villages, and actively tracked and monitored new imagery to determine if any violence had occurred. This imagery was paired with human rights organizations' documentation and eyewitness reports to ground truth the data, and also to provide a human aspect for the visitors of the site to relate to. ${ }^{4}$

This study used both high resolution and medium resolution satellite imagery to test three semiautomated remote sensing methods, in an attempt to identify useful tools to support eye-witness testimony and 
reports on human rights violations. As huts are routinely burned down during attacks on a village, particularly in Sudan, ${ }^{5}$ the number of huts and villages burned can be used to corroborate on-the-ground reports. As projects like Eyes on Darfur relied on manual examination of imagery, which is time consuming, a semiautomated method can speed up the analysis and expand areas that are monitored with limited resources. The Gereida region in Darfur, Sudan (Figures 1 and 2) was chosen as a study area for multiple reasons: Amnesty International and the American Association for the Advancement of Science (AAAS) published reports of villages being burned by Janjaweed horsemen between November of 2005 and February of 2006 around the town of Gereida; high resolution IKONOS imagery of the region was able to be acquired for both before and after the attacks occurred; and medium resolution Landsat ETM+ (Enhanced Thematic Mapper Plus) imagery before and after the attacks were available for the study region.

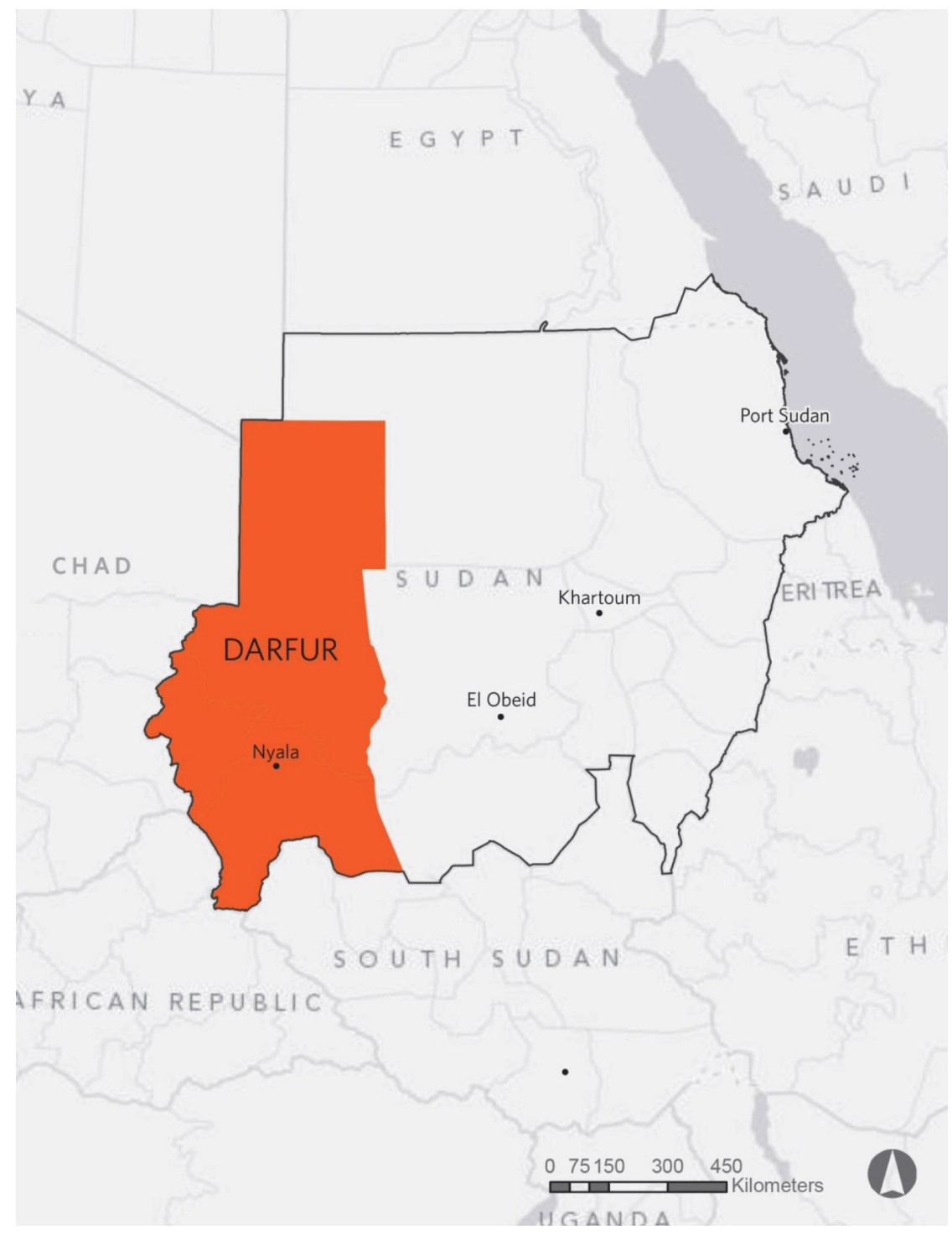

Figure 1. Map of Darfur and Sudan.

Data source: GADM database of Global Administrative Areas, January 2012. http://www.gadm.org/ (accessed August 25, 2013). Basemap: ESRI data and maps, 2012.

High resolution satellite imagery typically allows the viewer to discern objects (cars, houses, etc.), while medium resolution imagery is used for more regional examinations, such as identifying delineations between farmland and forested areas. Because high resolution imagery can "zoom in" more than medium 


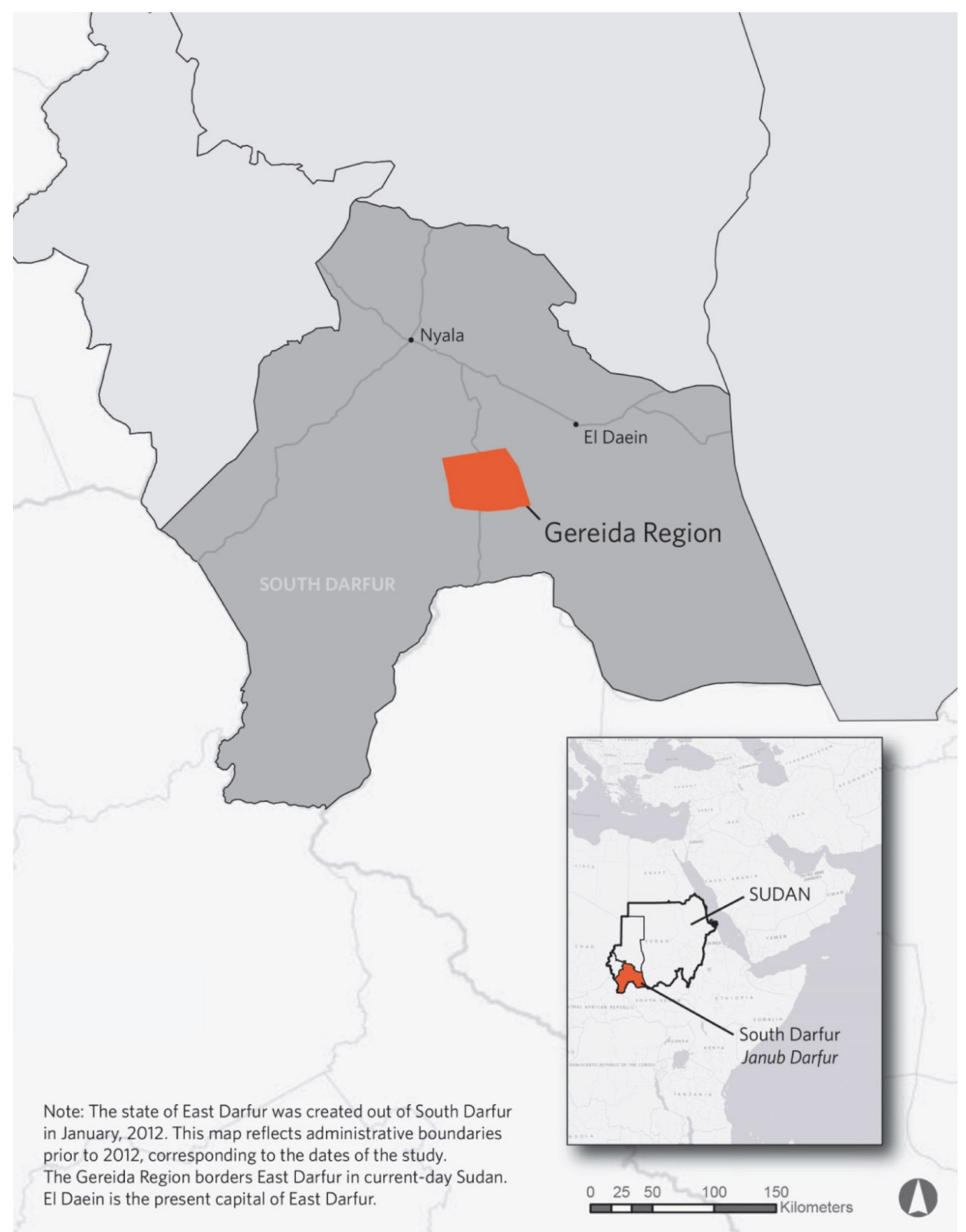

Figure 2. Map of Gereida Region.

Data source: GADM database of Global Administratvie Areas, January 2012. http://www.gadm.org/ (accessed August 25, 2013).

resolution, the area that is captured in a single image is smaller. The three remote sensing methods (Supervised Classification, Change Detection, and Feature Extraction) are semi-automated processes that can aid in the manual analysis of satellite imagery. These methods will be discussed more fully in this article. Data limitations occurred during this study causing some inconclusive results, but Feature Extraction emerged as a remote sensing method with positive results, indicating that it could be a useful tool to support eye-witness testimony gathered by human rights organizations (Table 1).

Remotely sensed data has been used as evidence of systemic human rights violations in Darfur, Sudan since 2003. The U.S. State Department's Humanitarian Information Unit used satellite imagery and Geographic Information Science (GIS) data to map where villages had been destroyed, allegedly by the Sudanese government, as well as other humanitarian issues that affected vulnerable populations like droughts and flooding. ${ }^{6}$ In addition to the higher resolution imagery like those used to identify destroyed villages, 
Table 1. Study results and conclusions overview.

\begin{tabular}{|c|c|c|c|}
\hline Method & Imagery used & Results & Conclusions \\
\hline \multirow{4}{*}{$\begin{array}{l}\text { Supervised } \\
\text { Classification }\end{array}$} & $\begin{array}{l}\text { IKONOS: Before } \\
\text { Attacks }\end{array}$ & $\begin{array}{l}\text { - Mahalanobis distance classifier } \\
\text { performed most accurately } \\
\text { - Village classification accuracy: } 78.54 \% \\
\text { - Total pixels classified as villages: } 36.53 \% \\
\text { (actual is } 3.27 \% \text { ) }\end{array}$ & $\begin{array}{l}\text { Inconclusive: } \\
\text { High rate of false positives }\end{array}$ \\
\hline & $\begin{array}{l}\text { IKONOS: After } \\
\text { Attacks }\end{array}$ & $\begin{array}{l}\text { - Mahalanobis distance classifier } \\
\text { performed most accurately } \\
\text { - Village classification accuracy: } 78.70 \% \\
\text { - Total pixels classified as burnt villages: } \\
12.93 \% \text { (actual is } 3.27 \% \text { ) }\end{array}$ & $\begin{array}{l}\text { Inconclusive: } \\
\text { High rate of false positives }\end{array}$ \\
\hline & $\begin{array}{l}\text { Landsat ETM+: } \\
\text { Before Attacks }\end{array}$ & $\begin{array}{l}\text { - Mahalanobis distance classifier } \\
\text { performed most accurately } \\
\text { - Village classification accuracy: } 45.47 \% \\
\text { - Total pixels classified as villages: } 27.62 \% \\
\text { (actual is } 0.55 \% \text { ) }\end{array}$ & $\begin{array}{l}\text { Inconclusive: } \\
\text { High rate of false positives } \\
\text { and low rate of accuracy }\end{array}$ \\
\hline & $\begin{array}{l}\text { Landsat ETM+: } \\
\text { After Attacks }\end{array}$ & $\begin{array}{l}\text { - Mahalanobis distance classifier } \\
\text { performed most accurately } \\
\text { - Intact village classification accuracy: } \\
71.44 \% \\
\text { - Burnt village classification accuracy: } \\
70.56 \% \\
\text { - Total pixels classified as villages: } 38.27 \% \\
\text { (actual is } 0.55 \% \text { ) }\end{array}$ & $\begin{array}{l}\text { Inconclusive: } \\
\text { High rate of false positives }\end{array}$ \\
\hline $\begin{array}{l}\text { Change } \\
\text { Detection }\end{array}$ & $\begin{array}{l}\text { IKONOS N/R/B } \\
\text { IKONOS N/R/G } \\
\text { Landsat } 4 / 3 / 2 \\
\text { Landsat BuRa }\end{array}$ & $\begin{array}{l}\text { - No clear visual patterns of change } \\
\text { resulted at any of the change thresholds }\end{array}$ & $\begin{array}{l}\text { Inconclusive: } \\
\text { No clear patterns }\end{array}$ \\
\hline \multirow{3}{*}{$\begin{array}{l}\text { Feature } \\
\text { Extraction }\end{array}$} & IKONOS N/R/B & $\begin{array}{l}\text { - Huts correctly identified: } 83.89 \% \\
\text { - False positive rate: } 34.09 \%\end{array}$ & $\begin{array}{c}\text { Inconclusive: } \\
\text { High accuracy, but also } \\
\text { high rate of false positives }\end{array}$ \\
\hline & IKONOS N/R/G & $\begin{array}{l}\text { - Huts correctly identified: } 47.43 \% \\
\text { - False positive rate: } 17.73 \%\end{array}$ & $\begin{array}{c}\text { Inconclusive: } \\
\text { Low accuracy, but low rate } \\
\text { of false positives }\end{array}$ \\
\hline & $\begin{array}{l}\text { Kernel Density } \\
\text { Estimation (IKONOS } \\
\text { N/R/G imagery) }\end{array}$ & $\begin{array}{l}\text { - Villages identified (no masking): } \\
43 / 77 \text { - } 55.84 \% \\
\text { - Villages identified (cloud masking): } \\
43 / 54 \text { - } 79.62 \%\end{array}$ & $\begin{array}{l}\text { Inconclusive, but positive: } \\
\text { No huts were extracted } \\
\text { in the clouded areas, but } \\
\text { a high rate of correctly } \\
\text { identified villages with KDE } \\
\text { after clouds were masked }\end{array}$ \\
\hline
\end{tabular}

medium resolution imagery can also be used to examine indirect results of violence, such as abandoned agricultural areas or the disappearance of livestock. Remote sensing methods can give researchers information that can be corroborated by on the ground accounts, or eyewitness reports from victims. Marx and Goward ${ }^{7}$ argue, however, that methods have changed very little since 2003, and very few organizations have used 
either high resolution imagery or medium resolution imagery, like MODIS (Moderate-Resolution Imaging Spectroradiometer, a sensor aboard the Terra and Aqua satellites), to track or detect fires that are violence related. Less than thirty academic articles are published on the subject in a given year, but since 2000, burned area detection has increased in use, based on a recent survey. ${ }^{8}$

High resolution satellite imagery has been studied as a way of counting affected population by the traces of systematic burning of huts in villages. Sulik and Edwards ${ }^{9}$ conducted a pilot project in 2010 to develop tools to identify villages and huts using high-resolution satellite imagery. The goal of the study was to determine the feasibility of accurately quantifying the number of huts for a given village using Feature Extraction methods, in this case for a village in Darfur, Sudan. Because of the limited access to Darfur, the estimates of death tolls from 2003 to late 2009 vary widely; 200,000-400,000 deaths, as reported by human rights organizations, down to the government of Sudan's official number of 9,000. This tool could be applied in the future to determine overall population in an area and population shifts as the result of loss/addition of dwellings, and is also suggested by the authors to be useful for advocates and policy-makers to circumvent the on-the-ground data collection limitations. ${ }^{10}$

The AAAS Geospatial Technology and Human Rights program provided Sulik and Edwards with standard Quickbird images of Jonjoma village in North Darfur, Sudan. A binary classification was conducted to separate the huts (and hut material) from all other features (such as soil or rock), which are considered background noise. A binary classification is a method in which the computer analyzes each pixel in the image and determines if it matches hut material or does not by using the spectral signatures of the pixels. A spectral signature is the amount of electromagnetic radiation (sunlight) reflected for a range of light wavelengths. The signature acts almost as a fingerprint for an object or material, and can be used to group pixels together by their shared properties (classification). The classification results were overlaid on pre-attack and postattack imagery to identify any changes. Some errors occurred, specifically tree shadows causing false positives because they have a similar spectral signature, size, and shape as thatched huts, making it difficult to distinguish between the two. The techniques discussed in the article are accurate enough to warrant further investigation (67\% identification accuracy), and would prove useful for most semi-desert landscapes where it is difficult to separate individual settlement structures from surrounding features. ${ }^{11}$

As new satellite technology increases spatial resolution, affordability, and faster access, scientific research points to a greater use of remote sensing and GIS in the future for the study of human rights issues. Mass violence and human rights violations have been major problems for the world populace, and the use of satellite imagery to monitor those issues by human rights organizations is growing based on the research and projects being published. Based on this research, searching for human rights violations by examining the loss of huts in rural areas is a useful tool to corroborate victim and eye-witness testimony. By using Feature Extraction methods and applying it in a timely fashion to other areas that have reports of violence, a faster and more thorough understanding of the incident or incidents can give governments and organizations more tools to respond to, and possibly forestall, deaths in situations of mass violence.

\section{Study Area}

The history of violence in Darfur has been well documented, ${ }^{12}$ and this article uses attacks that occurred in 2006 as a focus for the remote sensing methods being studied. This study concentrates on the area around the town of Gereida (Figure 2), approximately $100 \mathrm{~km}$ south of the Janub (South) Darfur capital of Nyala in Sudan. Amnesty International reported that on February 16, 2006, villages in the surrounding area around Gereida were attacked by the Janjaweed. Thirty-three people were killed, villages were looted, and over 1,500 heads of cattle and camels were stolen. The villages were inhabited by members of the Masalit tribe, a nonArab ethnic group that resides in Chad and Sudan, and part of the Darfuri rebel alliance. ${ }^{13}$ The Masalit live in a primarily agricultural society, growing peanuts, sorghum, millet, and other grains as both cash crops and subsistence. ${ }^{14}$ Between November 2005 and February 2006, over 300 people were killed in the region, with more than sixty villages burned. The number of internally displaced persons (IDPs) grew to nearly 90,000 , swelling Gereida with makeshift tents and shelters. ${ }^{15}$ As of 2010 , Gereida was the largest IDP camp in the world with over 100,000 displaced people. ${ }^{16}$ The town of Gereida and the IDP camp are immediately to the north of the area covered by the high resolution IKONOS satellite imagery in this study, and thus outside of the study area. Known villages in the high resolution imagery study area that were destroyed were Tigla, Arediba Araj, Umrokbi, Gubai, Umtraigo, Gandako, Hashaba, and Rajaj. ${ }^{17}$ Reports on villages around, and including, Tigla indicate that prior to the attacks in February 2006, 48 settled locations were counted. After the attacks, all 48 locations were destroyed, which had contained 1,660 structures. ${ }^{18}$ 


\section{Data and Methodology}

\section{Methods Overview}

Remote sensing is the process of acquiring and analyzing data without physically contacting the target, and in this article will refer to imagery collected by satellites. Three remote sensing methods (Supervised Classification, Change Detection, and Feature Extraction) were performed on imagery from both before the attacks (before imagery) in February 2006 and after the attacks (after imagery) to examine any useful trends that could be used by researchers when manually examining the satellite data (Figure 3 ). As illustrated in Figure 3, no huts exist after the attacks in the image on the right.

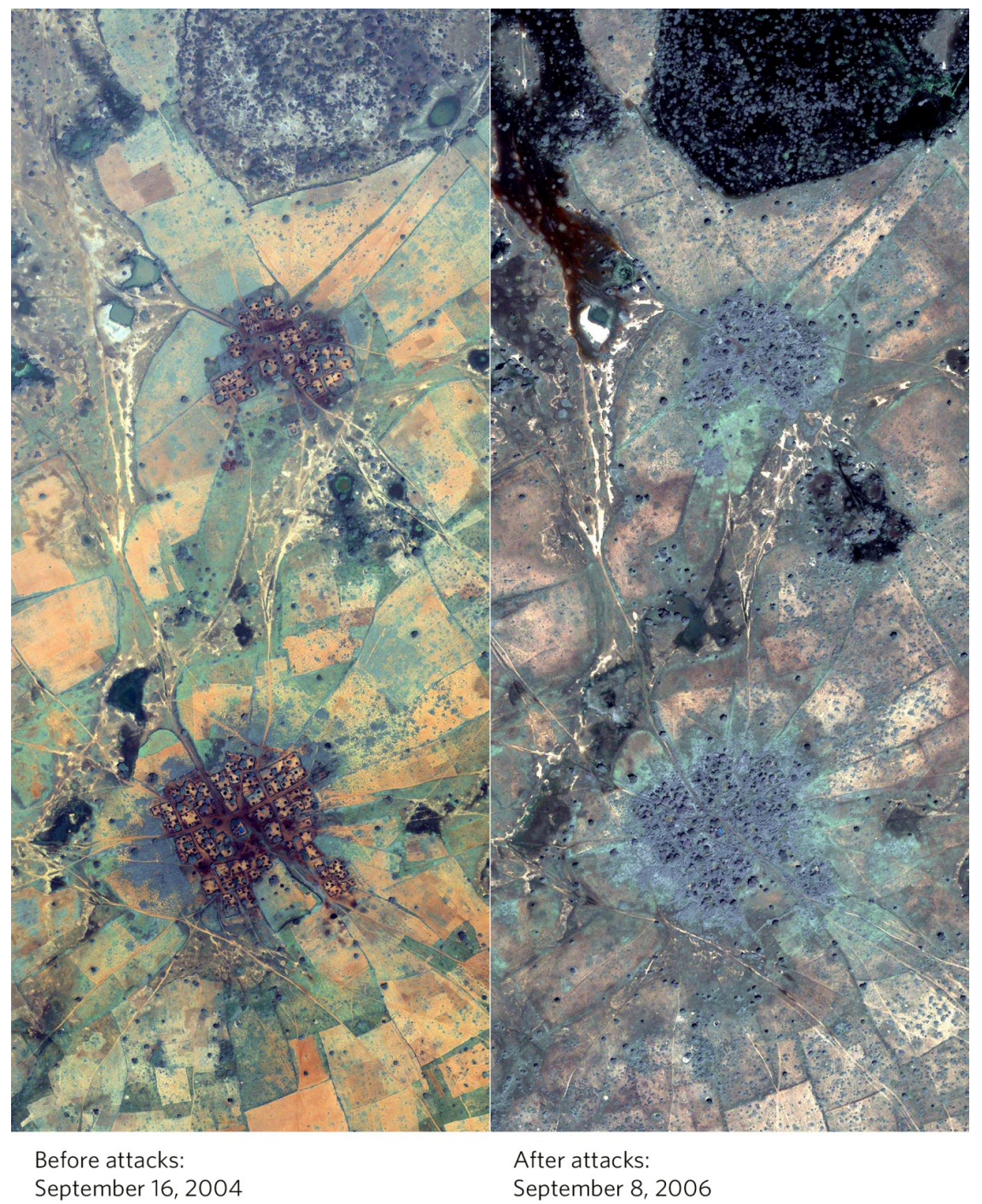

Figure 3. Satellite imagery of a village before and after the February 2006 attacks. Satellite imagery courtesy of the DigitalGlobe Foundation / GeoEye.

Satellite imagery is typically a digital file composed of millions of pixels, similar to images from a digital camera. There are two differences, however. Satellite imagery is georeferenced (tied to specific spots on earth using a coordinate system like latitude/longitude), and each pixel contains data for the sunlight reflected from objects in different wavelengths - from visible light like red or blue wavelengths, to invisible light like near infrared or ultraviolet wavelengths. Every material reflects light a certain way, and the combination of data from different wavelengths can create a spectral signature for that material. The data for each wavelength spectrum (blue light, near infrared light, etc.) is stored in separate files called bands, and when these bands are combined, can be used to create stacked imagery. This stacked imagery can be created to show the actual 
true color of an area (RGB), or create false-color imagery to highlight differences in another wavelength (Near Infrared/Red/Blue is often used to monitor vegetation, as infrared wavelengths are highly reflected by living vegetation). Each pixel in georeferenced imagery represents a distance that can be used in analysis. Satellite imagery collected using a sensor that has a 0.5 meter spatial resolution, for example, means that each pixel is a square 0.5 meters wide. Medium resolution imagery has a coarser spatial resolution (less detail), and a sensor like Landsat ETM+ has a resolution of 30 meters.

Supervised Classification is a method to classify pixels by their spectral signature. As each material (soil, water, etc.) has a spectral signature, pixels that share similar spectral signatures can be classified by a remote sensing program. In Supervised Classification, the user provides sample signatures that the program should classify each pixel into by highlighting areas as training sites, while Unsupervised Classification lets the computer group pixels by shared signatures automatically and the user decides what those classes are after the fact. Supervised Classification is useful for identifying classes in the imagery like forests, water, and urban area. Problems can arise, however, when lower spatial resolution imagery is used, because there is a higher probability that pixels will combine two different spectral signatures, making classification difficult.

Change Detection typically takes two sets of classified imagery (either supervised or unsupervised) from the same geographic area and measures the number of pixel changes from one class to another. This can be very useful in identifying what percentage of forest has been converted to agriculture, or where flooding has occurred, for example. Change Detection can also be used for unclassified imagery by identifying the change in value of the spectral signatures for each specific pixel. For example, burned material will have the spectral values decrease, and Change Detection can identify those changes if the decrease is large enough.

Feature Extraction is a method that combines identification of spectral signatures with an algorithm to detect spatial patterns, such as rectangular objects or linear roadways. This method is often used for detecting buildings and roads as they are fairly homogenous spectrally and uniformly geometric, and because Feature Extraction can be much faster than manually tracing a roadway or building outline by hand. Users develop training sites, which tell the program what to look for, both spatially and spectrally. In the case of this study, the training sites were created by manually outlining a subset of huts in the study region using circles. The output data in Feature Extraction is different than in either Supervised Classification or Change Detection. In the two latter methods, the output is raster data, which consist of pixels. In Feature Extraction the output is vector data, which is data composed of points, lines, and polygons. These vector data can be easily imported into a GIS program and analyzed using a variety of tools. In the case of this study, point data were used to demarcate the huts, and a kernel density function was performed on the vector output data.

A kernel density function is used to visualize the frequency of objects in a specific area, and is also known as a hotspot map. The function counts the number of points surrounding each point by calculating the distance from the target point to every point in the study area (using a mathematical method called inverse distance weighting). The points are weighted by the number and distance of other points to it (with a nearer and more densely concentration of points giving more weight), and a color map is created using the weighted averages. The darker areas have the highest concentration of points, while the lighter areas have the lowest concentration. The outcome is a map that visualizes where the highest concentrations of points are located, allowing a viewer to quickly identify areas that are densest.

Supervised Classification, Change Detection, and Feature Extraction were used in this study in an attempt to identify useful methods for verifying reports of burned villages. Villages in Darfur are composed of conical huts (also known as ghotiyas or tukuls) in small compounds. The huts have walls constructed with mud bricks and a thatched roof made from local grasses. ${ }^{19}$ Fences surrounding the compounds are also made of local grasses and branches, and enclose a large residential hut surrounded by multiple smaller huts that hold large ceramic pots used for food and grain storage. Small corrals are sometimes built inside the fences using local wood to hold livestock. Dirt pathways and roads are visible in the satellite imagery connecting the villages and hut compounds, and can be used to locate villages by tracing paths between them. As huts are burned during an attack, the charred remains form a black circle of ash where the walls once stood, while ceramic pots are often smashed to prevent repopulation. ${ }^{20}$

Data

Three types of satellite imagery were acquired for this study. The high resolution imagery used to identify individual huts was IKONOS imagery, which was acquired through the DigitalGlobe Foundation's Satellite Imagery Grant. ${ }^{21}$ The DigitalGlobe Foundation is a non-profit organization that awards imagery 
grants to researchers and students for use in a variety of studies including climate change research, forestry management, national security, and human rights. The DigitalGlobe Foundation graciously awarded satellite imagery for this study for an area of villages in the Gereida region, corresponding to dates from before and after the February 2006 attacks. High resolution OrbView 3 imagery, which was used as reference imagery for the methods, and medium resolution Landsat ETM+ imagery were acquired through the United States Geological Survey's (USGS) EarthExplorer. The Landsat ETM+ imagery was used for Supervised Classification and Change Detection, as the spatial resolution was too low to identify huts using Feature Extraction.

Observation satellites like IKONOS have sensors that collect data across multiple wavelengths and are broken into bands. Each band focuses on a certain wavelength spectrum (such as blue visible light or near infrared light). These different bands have different spatial resolutions, depending on the strength of the light it is capturing. Panchromatic bands capture all visible light, producing black and white imagery, and tend to have higher spatial resolutions than other bands. The multispectral bands (Near Infrared, Red, Green, and Blue bands) can be combined with the panchromatic band to increase the spatial resolution, resulting in pansharpened imagery. This article will abbreviate near infrared bands as $\mathrm{N}$, red bands as R, green bands as $\mathrm{G}$, and blue bands as $\mathrm{B}$.

All satellite imagery was acquired in the GeoTiff format, and was processed in ERDAS IMAGINE and converted to .img format. Supervised Classification, Change Detection, and Feature Extraction were performed in ERDAS IMAGINE, and vector files were exported into ArcGIS and analyzed. The normalized burn ratio imagery was created using the image calculator in Idrisi Selva, and exported into ERDAS IMAGINE. Feature Extraction was performed in ERDAS IMAGINE using the Feature Analyst module by Overwatch Systems.

The high resolution IKONOS panchromatic (black and white) imagery has 0.82 meter resolution, with a multispectral resolution of 3.2 meters. The panchromatic and multispectral imagery can be merged to create 1 meter resolution pan-sharpened data. The satellite has a swath width of 11.3 kilometers, which is the width of the area collected in the imagery. Four bands were used in this study; Blue $(0.445-0.516 \mu \mathrm{m})$, Green $(0.506-$ $0.595 \mu \mathrm{m})$, Red $(0.632-0.698 \mu \mathrm{m})$, and Near Infrared $(0.757-0.853 \mu \mathrm{m}){ }^{22}$ Three dates were acquired for the study area, two dates before the attacks (September 16, 2004 and September 24, 2004), and one date after the attacks (September 8, 2006). Pan-sharpened false-color infrared (N/R/G and N/R/B) imagery was created, as well as pan-sharpened true-color imagery $(\mathrm{R} / \mathrm{G} / \mathrm{B})$.

High resolution Orbview 3 imagery was used as reference in identifying features in the Landsat ETM+ imagery. The Orbview 3 imagery was obtained through the USGS EarthExplorer for dates before and after the attacks. Five panchromatic images were acquired with 1 meter resolution for April 29, 2005 (two images) and December 27, 2006 (three images). The data for both dates were adjacent to each other and were in the region covered by the Landsat ETM+ imagery. As the IKONOS after attack imagery did not contain any existing villages or huts, the Orbview 3 imagery was used to identify villages that existed in late 2006. These villages were used to create training sites for the Supervised Classification of the Landsat ETM+ imagery, as well as reference for analyzing the Change Detection results. The Orbview 3 imagery did not show any signs of genocide or burned villages after the attacks in February 2006. Orbview 3 imagery was not used for the remote sensing methods testing.

Landsat ETM+ imagery was collected for before and after the attacks, but with a larger period of time between them. The dates collected were for October 17, 2000 and August 31, 2006. Landsat ETM+ has a swath width of 183 kilometers, and eight bands were available, ranging from $15 \mathrm{~m}-60 \mathrm{~m}$ resolution. The panchromatic band, band 8, has a 15 meter resolution, while bands $1(\mathrm{~B}), 2(\mathrm{G}), 3(\mathrm{R}), 4(\mathrm{~N})$, and 7 (MidInfrared) have spatial resolutions of 30 meters. ${ }^{23}$ Multiple band combinations were created, all pan-sharpened to 15 meter resolution using band 7. False-color infrared imagery was created using bands 4, 3, and 2 (N/R/G). A second false-color infrared image was created using a combination described by Sulik and Edwards ${ }^{24}$ that stacked bands 4,3 , and $1(\mathrm{~N} / \mathrm{R} / \mathrm{B})$. A normalized burn ratio (BuRa) image, taken from research performed by Key and Benson ${ }^{25}$ was created using an image calculation in Idrisi: $\mathrm{BuRa}=(\mathrm{B} 4-\mathrm{B} 7) /(\mathrm{B} 4+\mathrm{B} 7)$. These bands show the greatest sensitivity to fire and burn effects, as band 7 increases with fire damage, and band 4 decreases. ${ }^{26}$

Landsat 7, the satellite that collects the Landsat ETM+ imagery, had its Scan Line Corrector (SLC) fail in May of 2003, resulting in a zig-zag type pattern of the imagery, and the post-collection correction results in the duplication of data in portions of the imaged area. ${ }^{27}$ Approximately 22 percent of a Landsat ETM+ scene will be lost due to the SLC failure, with the errors the most pronounced along the edges of the scene. ${ }^{28}$ The Gereida region and the IKONOS imagery is located in the southwest corner of the Landsat imagery, and therefore the study area for the 2006 Landsat ETM+ imagery has errors associated with the SLC failure. 
The Landsat ETM+ imagery that was acquired was corrected, however errors persisted and the 2006 Landsat ETM+ imagery is not a completely accurate representation of the area, even though it is still useful.

One problem that was encountered early in the study was that the high resolution IKONOS imagery had clearly defined villages and huts in the before imagery, while after the attacks all villages and huts in the study region were burned, leaving no control data (remaining villages) to examine before and after changes. Lower resolution Landsat ETM+ imagery was acquired to attempt to compensate for the lack of intact villages in the IKONOS imagery. As the Landsat ETM+ data encompassed the IKONOS region, villages identified in the IKONOS imagery were used to find pixels with the same spectral signatures in the Landsat ETM+ imagery, as well as classifying pixels that had similar spectral signatures to burned villages in the after imagery. Orbview 3 imagery was also used in a similar fashion to identify villages, as well as shrubs, grasslands, and other features, in the Landsat ETM+ imagery (Figure 4). However, the Orbview 3 imagery did not contain any burned villages for either date collected, and was only used as verification data to compare the before and after classified Landsat ETM+ imagery.

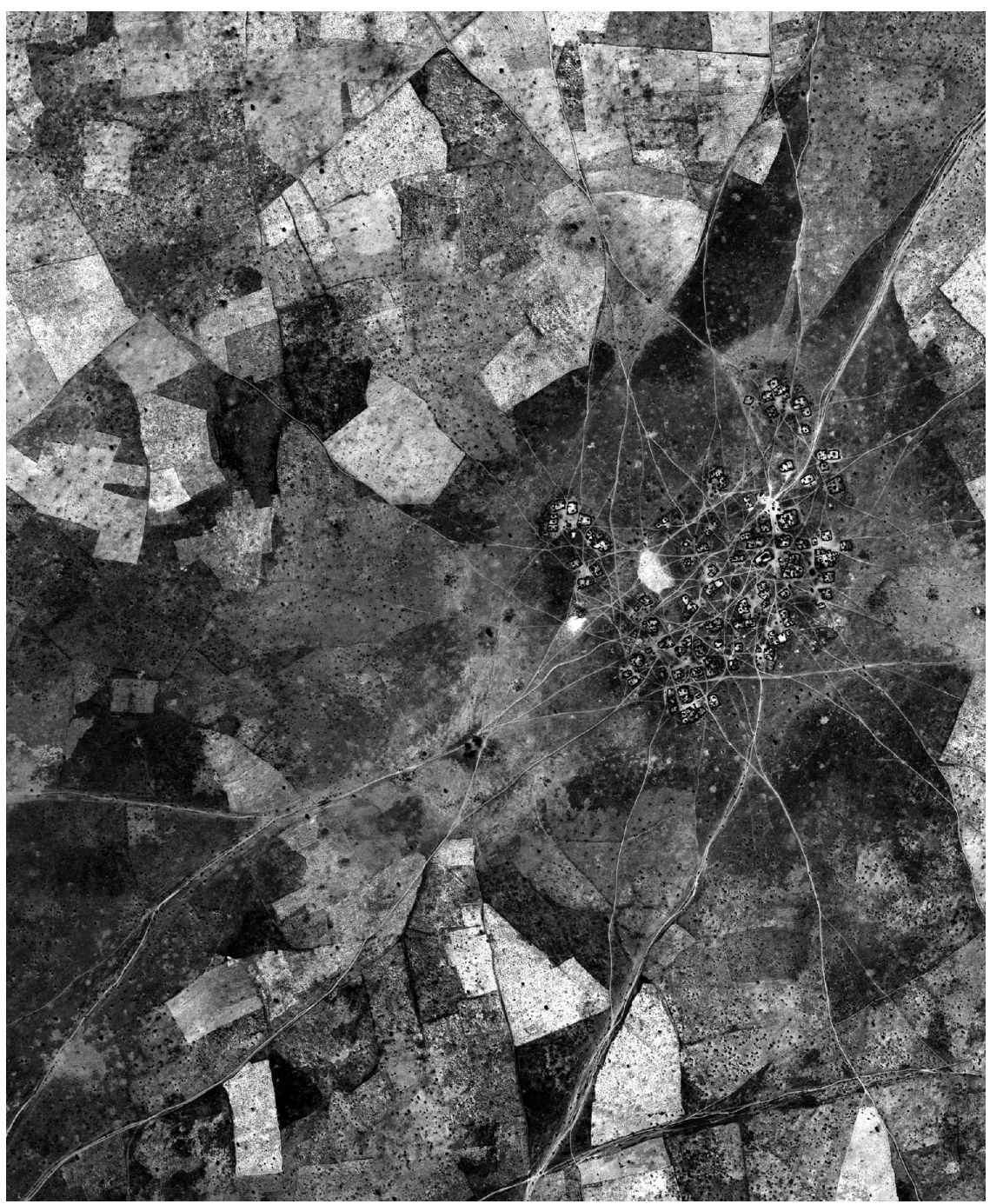

Figure 4. Orbview 3 imagery showing a village to the east of the study area. Satellite imagery courtesy of the USGS EarthExplorer.

As this study was intended to determine if there were any useful semi-automatic ways to analyze burned villages to assist manual examination of imagery, no masking of clouds or other objects took place. Clouds can cover portions or all of an area in satellite imagery, and a typical method to reduce the distorted or missing data under cloud cover is to segment the imagery into clouded and cloud free 
portions, called masking. Some imagery, particularly the IKONOS imagery from before the attacks, had cloud cover (September 16, 2004 imagery had 22\% cloud cover, and September 24, 2004 imagery had 17\% cloud cover). The intention of not masking cloud cover was to ascertain if any useful information could be extracted from those areas for use in a manual analysis, as some villages were visible beneath the cloud cover upon visual inspection.

\section{Technical Details}

Supervised Classification uses the spectral signatures of pixels in the imagery to classify them into feature classes determined by the user. The goal of the Supervised Classification method in this article was to identify villages in the study area, both intact and burned. Once the villages have been identified in both before and after attack imagery, theoretically, they can be counted and paired up with human rights organization reports to corroborate violations. Multiple Supervised Classifications were conducted on both the high resolution (IKONOS) and lower resolution imagery (Landsat ETM+) using ERDAS IMAGINE. False-color infrared imagery was created for both the IKONOS and Landsat ETM+ imagery by stacking the near-infrared, red and green bands (bands 4,3 , and 2). The resulting multispectral imagery was pan-sharpened using the panchromatic band (band 8 in the case of Landsat ETM+) in a resolution merge in ERDAS IMAGINE, resulting in the IKONOS imagery having a spatial resolution of 1 meter, and the Landsat ETM+ imagery having a spatial resolution of 15 meters.

Training sites were developed by hand for different landscape features, and were only created for clearly defined features. Google Earth satellite imagery, photography from Google Earth, and Orbview 3 imagery were used to help identify and confirm features. The features used were agriculture, arroyo (dry river beds), grassland, open water (rivers, lakes, and ponds), rocks (in the northern section of the Landsat ETM+ outcrop existed), trees and brush, and villages (both intact and burned).

The after imagery included existing villages and burned villages, of which the Landsat ETM+ imagery had both, while the IKONOS imagery only had burned villages. In addition, individual huts were identified for the IKONOS imagery to attempt high resolution classification. The total number of huts identified was 3,190 in 77 villages. The definition of a village in this study was a clustering of three or more compounds, which consisted of huts surrounded by fencing. Huts were identified based on previous articles' descriptions, in particular Sulik and Edwards 2010 article on Feature Extraction in Darfur. ${ }^{29}$ Classification training sites were created for the before and after attack imagery using separate signatures, because features had changed between the time periods and different signatures were necessary for the IKONOS and Landsat ETM+ imagery.

Three classification techniques were performed on the imagery using the training sites: Gaussian Maximum Likelihood, Minimum Distance to means, and Mahalanobis Distance. The maximum likelihood classifier uses variance and covariance to identify the statistical probability of a given pixel being part of a predetermined class..$^{30}$ Maximum likelihood is considered the most powerful method, and one of the most widely used. ${ }^{31}$ Minimum distance is a method that, by using a scatterplot of all pixels, identifies the minimum distance between a given pixel and the mean of a class of predetermined pixels. Because of the simplicity, minimum distance fails to take into account variations in spectral signatures, and "is not widely used in applications where spectral classes are close to one another in the measurement space and have high variance". ${ }^{32}$ Regardless, this method was employed in this study as it is a commonly used tool and is accessible to researchers. Mahalanobis Distance classification is a method based on the minimum distance algorithm, but it uses a covariance matrix. It is regarded as the most accurate of the three principle classification methods in ERDAS IMAGINE and is most used for cluster analysis. ${ }^{33}$ One reason for the high accuracy rates is that it can filter out shadows in addition to classifying highly varied clusters of objects. ${ }^{34}$ As villages tend to be highly clustered, and trees and huts cast shadows depending on what time of day the imagery was taken, in theory the Mahalanobis distance classification would be ideal for identifying villages in a given area.

The three classification methods were performed using five sets of satellite imagery. Three IKONOS imagery sets were classified: September 16, 2004, September 24, 2004, and September 8, 2006. Two sets of Landsat ETM+ imagery were classified: October 17, 2000 and August 31, 2006. The three classification methods were performed on the false-color infrared imagery for all five sets of data, resulting in fifteen classification results. The results were saved as img files, and contingency matrices were calculated (Tables 2 , 3,4 , and 5). The contingency matrices show the number of pixels that are classified for each class, as well as 
percentages of pixels classified per class, and the accuracy of each class classification in relation to the training site pixels (i.e. what percentage of pixels in the training sites for each class were classified correctly). An analysis of the percentages in conjunction with the number of pixels was used to determine which classifier would be most useful to identify burned villages.

The remote sensing method of Change Detection is used to "discriminate areas of land cover change between dates of imaging". ${ }^{35}$ Typically, classified imagery (either resulting from Supervised Classification or Unsupervised Classification) from two time periods of the exact same study area is used to determine if pixels have changed classes, but this is dependent on the quality and accuracy of the classification methods. The Supervised Classification results in this study did not produce satisfactory data that would be beneficial for Change Detection. As a result, this study used stacked imagery that was not classified in any form, with multiple combinations of bands tested. The expectation in using the stacked imagery is that because villages can be identified by visual examination, and burned villages can be identified in the false-color infrared imagery in particular, the Change Detection method would be able to identify villages that have changed from "existing" to "destroyed" based on the dramatic change in pixel values. Both the IKONOS imagery and the Landsat ETM+ imagery were used to detect changes in the spectral signatures of the study area. The IKONOS Change Detection used the false-color N/R/G and N/R/B imagery. The Landsat Change Detection used false-color infrared imagery and the normalized burn ratio calculated imagery. Change Detection was performed between the before and after imagery, and vector outlines of the villages were layered on top for visual examination of the results. As this study is to find methods that aid in the manual examination of satellite imagery, patterns of change in and around villages were visually analyzed. Thresholds of change were used in an attempt to isolate villages or patterns in the data. The thresholds are percentages of change in the pixels' signature, either an increase or decrease in intensity per band. Both IKONOS and Landsat ETM+ imagery used thresholds of $5 \%$ to $50 \%$, with an increase incrementally by $5 \%$.

Feature Extraction is a method to discern features by a combination of shape, size, and spectral signature. This method is very useful for identifying buildings and streets due to their geometric patterns. This study used Feature Extraction in an attempt to isolate individual huts, which when compared between before and after imagery, would give an estimate on how many huts were burned and where. The problem with the imagery acquired is that all huts in the imagery from after the attacks took place had been burned and none survived, based on a visual analysis. This method explored Feature Extraction in the before imagery only, with the intention that the method could be applied to other regions in the area in future studies if they have both surviving and destroyed villages or huts in the same data.

Feature Extraction was performed using Feature Analyst for IMAGINE. The IKONOS imagery was used for Feature Extraction, as the Landsat ETM+ imagery was too coarse to identify individual huts. Two pansharpened stacked images (N/R/G and N/R/B) were used for the Feature Extraction. The second combination (N/R/B) was used in another Feature Extraction study that resulted in satisfactory results. ${ }^{36}$

Using the Feature Analyst vector tools, polygons were placed over randomly picked huts across the image, with the polygons being created in a circular fashion to match the borders of the huts. 98 huts were recorded in the feature class. Parameters were set in the Learning module of the program, which instructs the program as to what shape the target features are. The closest parameter to the size and shape of the huts was Bull's-eye 1 , which was circular with a pattern width of 5 cells. The Feature Analyst manual recommends the Bull's-eye 1 for small man-made features. The extraction process was started, and two sessions of hierarchal learning were conducted to teach the Feature Extraction module the correct and incorrect polygons that represent the huts. The Remove Clutter by Shape function was performed on correctly and incorrectly identified features, with the perimeter, area, and compactness parameters selected. An aggregate function was first performed, which eliminated any polygons with less than 15 pixels, but this resulted in poor outcomes due to large areas of pixels being identified as huts that bear no relation in shape to the training polygons, therefore the shape function was used. This function resulted in a series of polygons, which were converted to points in the program, and the shapefiles were brought into Esri ArcMap for analysis.

Additionally, the hand count of huts in the study area used in the classification method was utilized for Feature Extraction, with a total of 3,190 individual huts. Polygons were created to count a subset of these huts in two villages, and an outline of all villages in the study area, resulting in a total of 77 villages. These data were imported into ArcMap for analysis, and comparison counts between the extracted data and the hand-counted data were performed. 


\section{Results}

Supervised Classification Results

Before Attacks Imagery Classification: IKONOS (2004 09 16)

The Supervised Classification method performed on the IKONOS imagery from before the attacks delivered inconclusive results. The Supervised Classification method using the village signature with the highest accuracy rate was the Mahalanobis Distance classification (Table 2). Table 2 compares the percentage of pixels accurately classified with manually identified pixels. Values other than total pixel counts are percentages. Dark green values signify high accuracy (above 80\%). Light green values signify medium accuracy (between $50 \%$ and $80 \%$ ). Orange values signify low accuracy (less than 50\%). Percentage of Total indicates the number of pixels in the image classified compared to all pixels. For pixels identified as villages, a high percentage is a poor result as villages constitute a very small percentage of the overall study area. In these data, the percentage of total pixels identified as villages is $36.52 \%$. Classified pixels identified as villages were manually identified as agriculture $30.83 \%$ of the time, which is a poor result.

Table 2. Supervised Classification accuracy results for IKONOS imagery from before the September 16, 2004 attacks.

\begin{tabular}{|c|c|c|c|c|c|}
\hline & \multicolumn{4}{|c|}{ Manually identified pixels } \\
\hline & & Villages & Agriculture & Trees and Shrubs & Water \\
\hline & Villages & 78.54 & 30.83 & 22.07 & 11.01 \\
\hline$\underline{x}$ & Agriculture & 6.6 & 67.58 & 0.73 & 0.36 \\
\hline वृ & \begin{tabular}{|l} 
Trees and Shrubs \\
\end{tabular} & 1.93 & 0.14 & 34.91 & 2.99 \\
\hline 娄 & Water & 0.53 & 0.01 & 1.35 & 74.39 \\
\hline 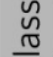 & Arroyo & 12.4 & 1.44 & 40.94 & 11.25 \\
\hline $\bar{U}$ & Total pixel count & 156502 & 751306 & 14347 & 22284 \\
\hline
\end{tabular}

\begin{tabular}{|c|c|c|c|c|}
\hline & \multicolumn{3}{|c|}{ Manually identified pixels } \\
\hline & & Arroyo & Total pixel count & Percentage of Total \\
\hline \multirow{6}{*}{ 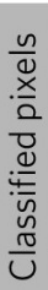 } & Villages & 32.65 & 488920 & $36.52 \%$ \\
\hline & Agriculture & 9.11 & 554148 & $41.39 \%$ \\
\hline & Trees and Shrubs & 4.01 & 25583 & $1.91 \%$ \\
\hline & Water & 14.44 & 74616 & $5.57 \%$ \\
\hline & Arroyo & 39.78 & 195485 & $14.60 \%$ \\
\hline & Total pixel count & 394313 & 1338752 & $100.00 \%$ \\
\hline
\end{tabular}

Mahalanobis Distance is a classifier that performs best on highly clustered objects, and was expected to have the highest accuracy among the three classifiers. The villages were accurately classified with a rate of $78.54 \%$, with the majority of the other pixels originally trained as villages being classified as arroyo. All of the Supervised Classifications had problems with the clouds in all of the IKONOS imagery, as a cloud mask was not applied. The downside of this classification, and all others performed on this imagery is that a large amount of pixels in the entire image were classified as villages (36.52\%). The IKONOS imagery, when manually counted, had $11.69 \%$ of pixels classified as villages, while the Landsat ETM+ imagery had $3.27 \%$ pixels classified as villages. This resulted in an unsuccessful classification when paired with a visual analysis of the imagery to determine if villages could be identified (Figure 5). In addition to villages being used as training pixels, another classification signature was created using individual huts, and a binary classification (a method in which each pixel is classified as one of two classes) and multi-feature classification were performed. Both of these performed poorly, as the accuracy rate was satisfactory (97.18\% for multi-feature and $84.62 \%$ for binary), but a high percentage of pixels in the entire image were classified as huts (10.21\% in the multi-feature and $41.78 \%$ in the binary classifications) which is not useful in identifying villages (Figure 6). The other IKONOS data set from 2004 (September 24, 2004) had similar results. 


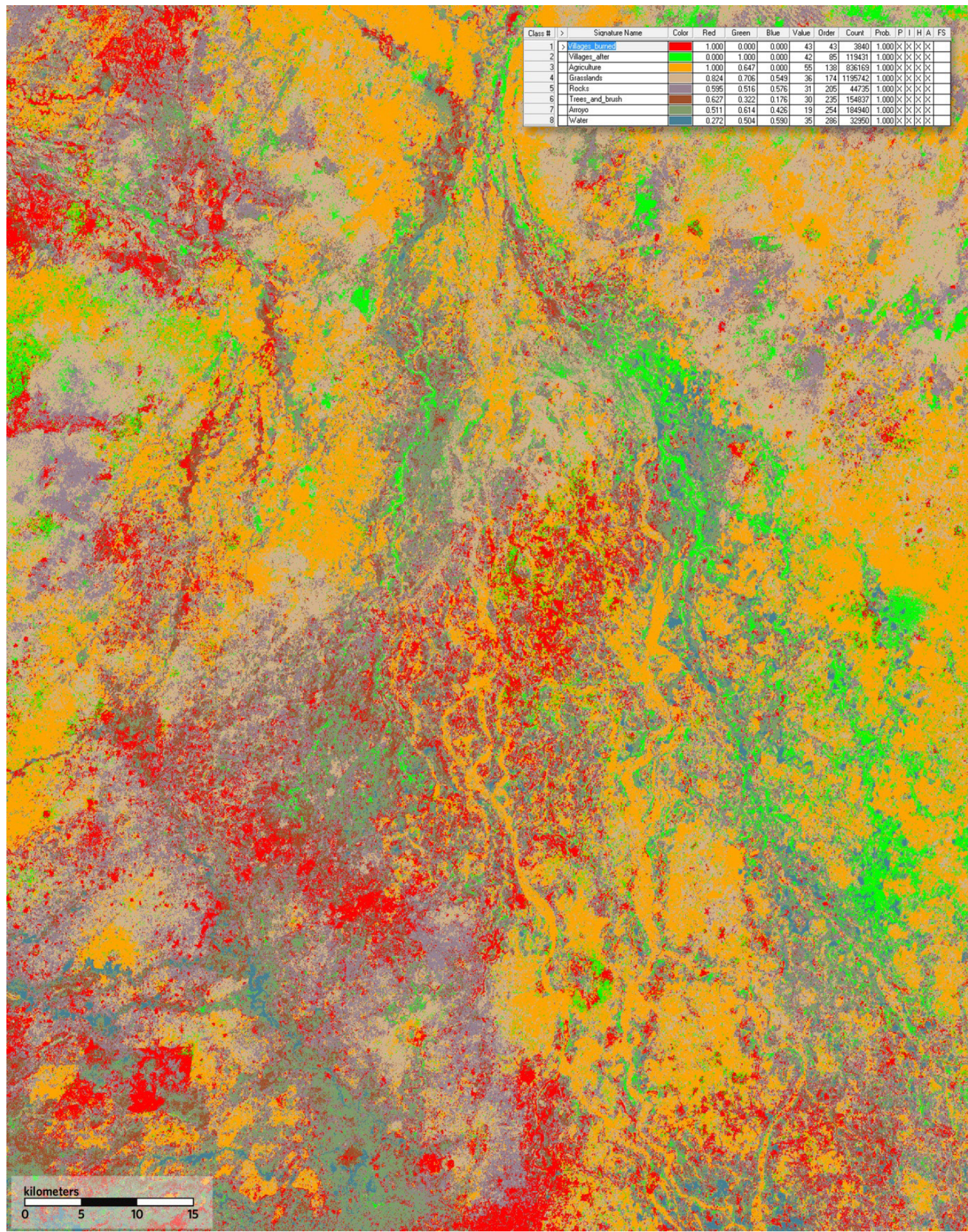

Figure 5. Supervised Classification results.

Satellite imagery courtesy of the DigitalGlobe Foundation / GeoEye. 


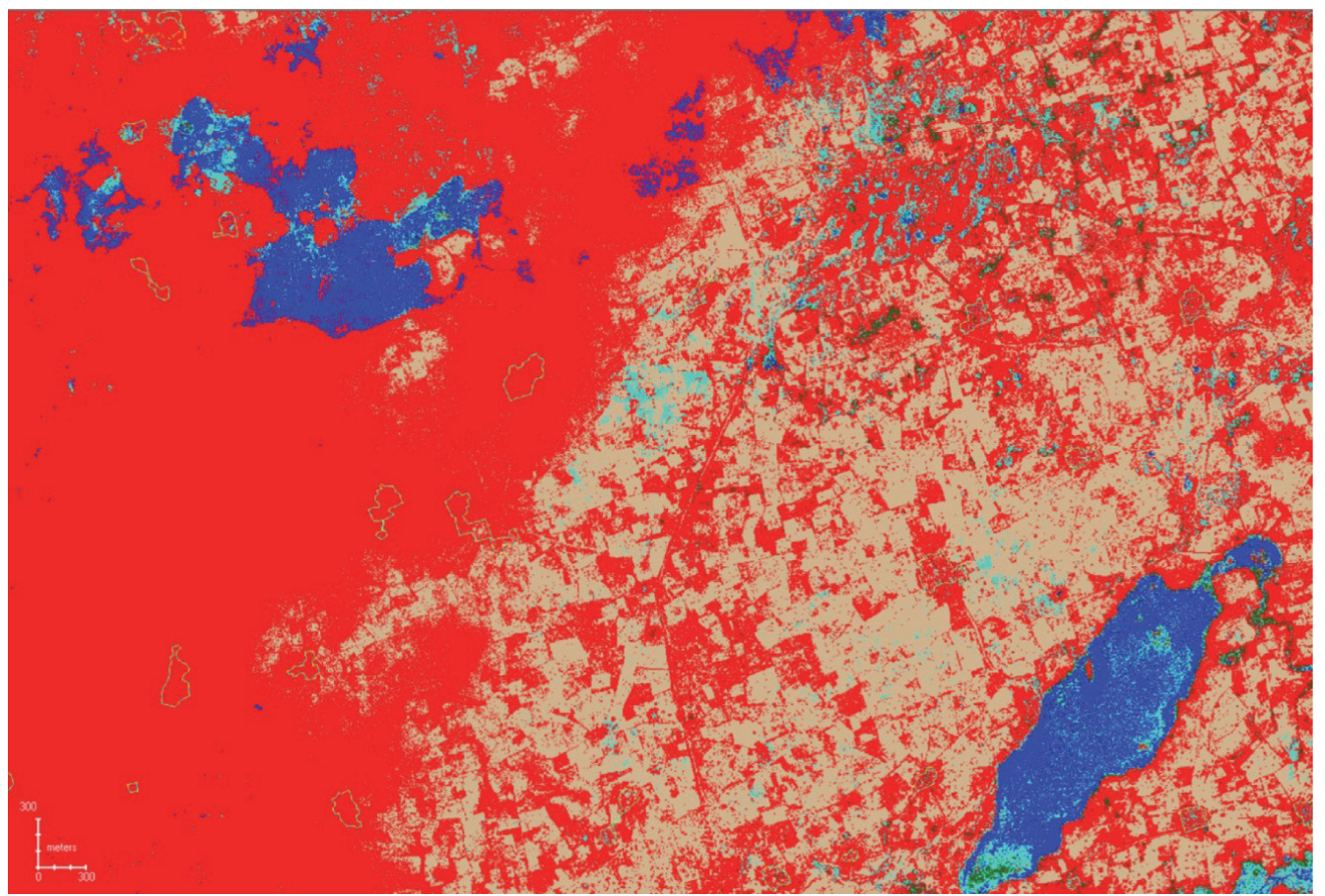

Figure 6. Supervised Classification results - IKONOS imagery before attacks. Satellite imagery courtesy of the DigitalGlobe Foundation / GeoEye.

\section{After Attacks Imagery Classification: IKONOS (2006 09 08)}

The Supervised Classification method performed on the IKONOS imagery from after the attacks delivered inconclusive results. The classification that performed with the highest accuracy rate among the three was maximum likelihood (Table 3). Table 3 compares the percentage of pixels accurately classified with manually identified pixels. Values other than total pixel counts are percentages. Dark green values signify high

Table 3. Supervised Classification accuracy results for IKONOS imagery from after the September 8, 2006 attacks.

\begin{tabular}{|c|c|c|c|c|c|}
\hline & \multicolumn{4}{|c|}{ Manually identified pixels } \\
\hline & & Burned Villages & Agriculture & Trees and Shrubs & Grassland \\
\hline \multirow{7}{*}{ 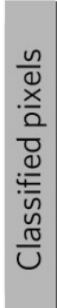 } & Burned Villages & 78.7 & 2.14 & 16.38 & 0.73 \\
\hline & Agriculture & 0.6 & 78.05 & 1.53 & 4.25 \\
\hline & Trees and Shrubs & 16.53 & 3.44 & 62.15 & 9.77 \\
\hline & Grassland & 1.51 & 12.57 & 6.15 & 79.76 \\
\hline & Water & 0.34 & 0.06 & 0.81 & 0.01 \\
\hline & Arroyo & 2.32 & 3.74 & 12.98 & 5.48 \\
\hline & Total pixel count & 1134946 & 4488366 & 756508 & 852859 \\
\hline
\end{tabular}

\begin{tabular}{|c|c|c|c|c|c|}
\hline & \multicolumn{4}{|c|}{ Manually identified pixels } \\
\hline & & Water & Arroyo & Total pixel count & Percentage of Total \\
\hline \multirow{7}{*}{ 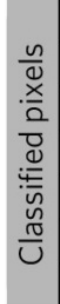 } & Burned Villages & 0.01 & 2.98 & 1173611 & $12.93 \%$ \\
\hline & Agriculture & 0.17 & 0.89 & 3574086 & $39.37 \%$ \\
\hline & Trees and Shrubs & 0.48 & 23.78 & 1328047 & $14.63 \%$ \\
\hline & Grassland & 0 & 30.22 & 1857726 & $20.46 \%$ \\
\hline & Water & 98.33 & 1.7 & 70993 & $0.78 \%$ \\
\hline & Arroyo & 1.01 & 40.42 & 1074790 & $11.84 \%$ \\
\hline & Total pixel count & 27830 & 1818744 & 9079253 & $100.00 \%$ \\
\hline
\end{tabular}


accuracy (above 80\%). Light green values signify medium accuracy (between 50\% and 80\%). Orange values signify low accuracy (less than 50\%). Percentage of Total indicates the number of pixels in the image classified compared to all pixels. For pixels identified as villages, a high percentage is a poor result as villages constitute a very small percentage of the overall study area. In these data, the percentage of total pixels identified as burned villages is $12.93 \%$. Classified pixels identified as burned villages were manually identified as trees and shrubs $16.383 \%$ of the time. Pixels classified as water had a very high accuracy rate $-98.33 \%$.

A comparison between villages burned and villages that survived is not possible using these data, because all of the villages in this region were burned. The accuracy of burned villages in the classification was $78.7 \%$, with agriculture having an accuracy of $78.05 \%$ and grasslands with an accuracy of $79.76 \%$. All three classifications had high accuracy rates for water, with the maximum likelihood accuracy being 98.33\%. However, as is the problem with the classification of the IKONOS 2004_09_16 imagery, the percentage of the entire image being classified as villages is high (12.93\%), and a visual analysis confirms this as there are a large number of false positives if used to identify burned villages (Figure 7). The IKONOS imagery, when manually counted, had $11.69 \%$ of pixels classified as villages.

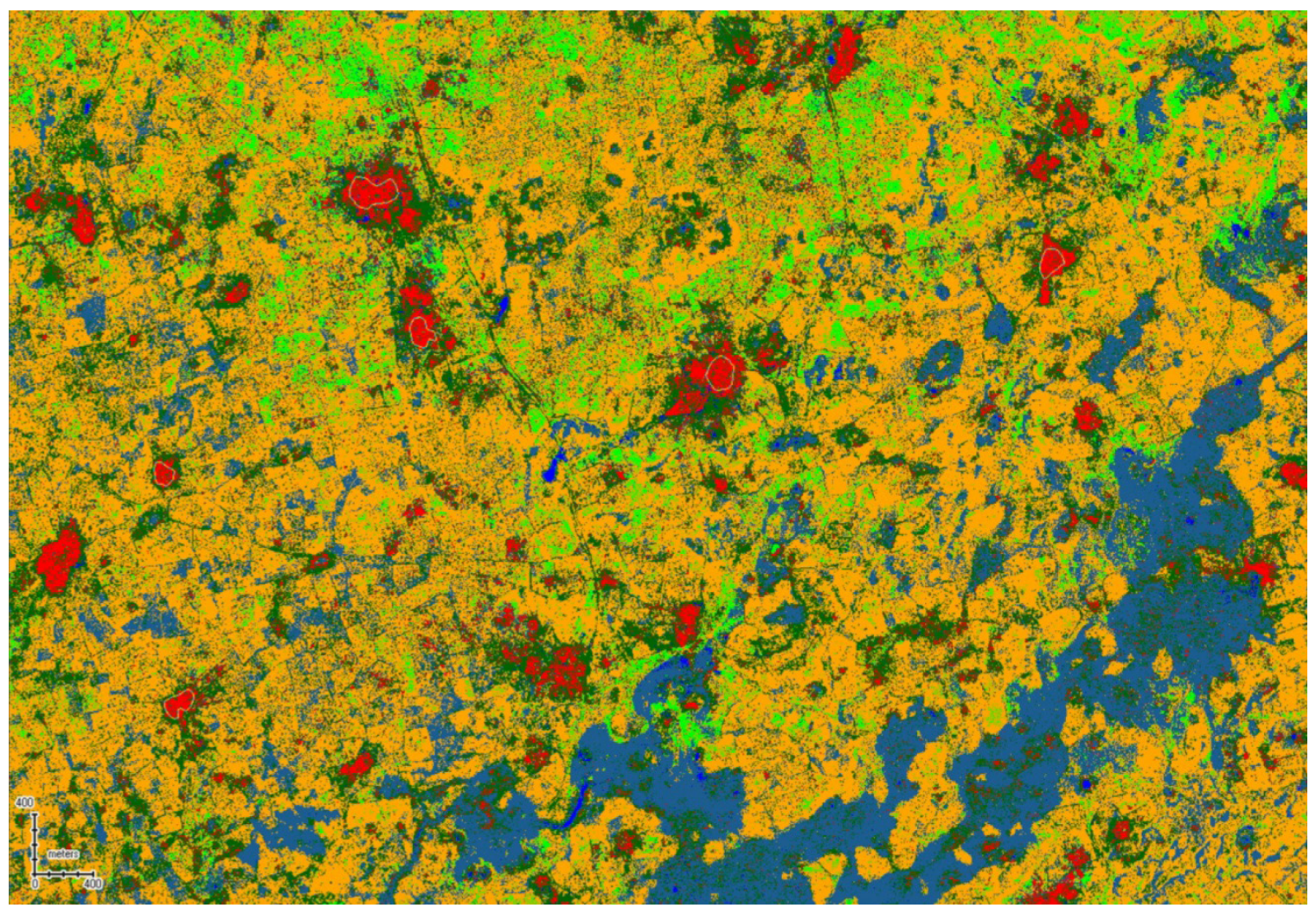

Figure 7. Supervised Classification results - IKONOS imagery after attacks.

Satellite imagery courtesy of the DigitalGlobe Foundation / GeoEye.

\section{Before Attacks Imagery Classification: Landsat ETM+ $\left.\begin{array}{lll}2000 & 10 & 17\end{array}\right)$}

The Supervised Classification method performed on the Landsat ETM+ imagery from before the attacks delivered inconclusive results. The most successful, in terms of accuracy rates, was the Mahalanobis distance classifier (Table 4). Village classification accuracy was $45.47 \%$, while agriculture had an accuracy of $84.61 \%$ and water had an accuracy of $90.02 \%$. Grassland classification performed poorly, with an accuracy of $27.62 \%$ (with the majority of the remaining pixels classified as village $-42.19 \%$ ). $27.62 \%$ of all pixels in the image were classified as village. The Landsat ETM+ imagery had 3.27\% pixels classified as villages. Similar results existed in with the other classification methods used (maximum likelihood and minimum distance). Visual analysis of the imagery resulted in no clear patterns relating to villages. Table 4, below, compares the percentage of pixels accurately classified with manually identified pixels. Values other than total pixel counts are percentages. Dark green values signify high accuracy (above 80\%). Light green values signify medium 
Table 4. Supervised Classification accuracy results for Landsat ETM+ imagery from before the October 17, 2000 attacks.

\begin{tabular}{|c|c|c|c|c|c|c|}
\hline & \multicolumn{5}{|c|}{ Manually identified pixels } \\
\hline & & Villages & Agriculture & Trees and Shrubs & Grassland & Open Water \\
\hline \multirow{8}{*}{ 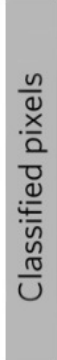 } & Villages & 45.47 & 9 & 11.07 & 42.19 & 0.27 \\
\hline & Agriculture & 20.27 & 84.61 & 2.34 & 14.74 & 0.61 \\
\hline & Trees and Shrubs & 7.43 & 1.46 & 40.09 & 4.68 & 2.9 \\
\hline & Grassland & 7.27 & 2.66 & 0.4 & 29.56 & 0 \\
\hline & Open Water & 0.21 & 0.03 & 9.93 & 0.01 & 90.02 \\
\hline & Arroyo & 7.79 & 1.68 & 27.89 & 5.19 & 5.88 \\
\hline & Rocks & 11.57 & 0.57 & 8.28 & 3.64 & 0.31 \\
\hline & Total pixel count & 111448 & 592385 & 420608 & 1827929 & 25207 \\
\hline
\end{tabular}

\begin{tabular}{|c|c|c|c|c|c|}
\hline & \multicolumn{4}{|c|}{ Manually identified pixels } \\
\hline & & Arroyo & Rocks & Total pixel count & Percentage of Total \\
\hline \multirow{8}{*}{ 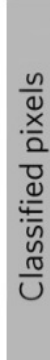 } & Villages & 3.27 & 12.82 & 939972 & $27.62 \%$ \\
\hline & Agriculture & 2.37 & 0.88 & 812589 & $23.87 \%$ \\
\hline & Trees and Shrubs & 13.96 & 11.14 & 329949 & $9.69 \%$ \\
\hline & Grassland & 0.04 & 0.38 & 566232 & $16.64 \%$ \\
\hline & Open Water & 13.95 & 4.57 & 120247 & $3.53 \%$ \\
\hline & Arroyo & 61.08 & 28.41 & 477856 & $14.04 \%$ \\
\hline & Rocks & 5.33 & 41.8 & 156705 & $4.60 \%$ \\
\hline & Total pixel count & 381234 & 44739 & 3403550 & $100.00 \%$ \\
\hline
\end{tabular}

accuracy (between 50\% and 80\%). Orange values signify low accuracy (less than 50\%). Percentage of Total indicates the number of pixels in the image classified compared to all pixels. For pixels identified as villages, a high percentage is a poor result as villages constitute a very small percentage of the overall study area. In these data, the percentage of total pixels identified as villages is $27.62 \%$. Classified pixels identified as villages were manually identified as grassland $42.19 \%$ of the time. Pixels classified as open water $(90.02 \%)$ and agriculture (84.61\%) had a high accuracy rate. Pixels identified as grassland had a low accuracy rate (29.56\%), as over $42 \%$ were identified as villages.

\section{After Attacks Imagery Classification: Landsat ETM+ (2006 08 31)}

The Supervised Classification method performed on the Landsat ETM+ imagery from before the attacks delivered inconclusive results. The classification with the highest accuracy was the Mahalanobis distance classifier (Table 5). Burnt villages classification had an accuracy of $71.44 \%$, and surviving villages had an accuracy of $70.56 \%$. However, as with all of the other classifications performed in this study, a large amount of false positives exists (all pixels classified as villages, either burned or surviving, accounted for $38.27 \%$ of the total image). Agriculture and grasslands had satisfactory accuracy, however, particularly with the maximum likelihood classification $(72.66 \%$ for agriculture and $66.33 \%$ for grasslands), and visual analysis shows that the classification generally matches the false-color infrared imagery. Areas around villages burned were typically grassland, while areas around surviving villages were typically agriculture. This could be useful in a future study in identifying abandoned villages, due to drought, famine, or violence, but only in a study examining past events. Visual analysis shows that the villages, especially cities such as El Daein close to the center of the study area, have a high concentration of agriculture radiating out, and the Supervised Classification method is able to capture this. Table 5 compares the percentage of pixels accurately classified with manually identified pixels. Values other than total pixel counts are percentages. Dark green values signify high accuracy (above 80\%). Light green values signify medium accuracy (between 50\% and 80\%). Orange values signify low accuracy (less than 50\%). Percentage of Total indicates the number of pixels in the image classified compared to all pixels. For pixels identified as villages, a high percentage is a poor result as villages constitute a very small percentage of the overall study area. 
Table 5. Supervised Classification accuracy results for Landsat ETM+ imagery from after the August 31, 2006 attacks.

\begin{tabular}{|c|c|c|c|c|c|c|}
\hline & \multicolumn{5}{|c|}{ Manually identified pixels } \\
\hline & & Villages before & Villages after & Agriculture & Grassland & Rocks \\
\hline \multirow{9}{*}{ 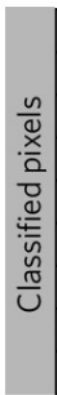 } & Villages before & 71.44 & 2.56 & 1.22 & 5.34 & 5.72 \\
\hline & Villages after & 2.79 & 70.56 & 16.73 & 52.46 & 9.76 \\
\hline & Agriculture & 0.89 & 14.33 & 72.99 & 10.88 & 0.44 \\
\hline & Grassland & 0.29 & 3.5 & 3.92 & 12.4 & 1.31 \\
\hline & Rocks & 2.84 & 1.75 & 0.38 & 5.85 & 31.04 \\
\hline & Trees \& Shrubs & 5.91 & 1.14 & 1.32 & 3.3 & 11.63 \\
\hline & Arroyo & 15.84 & 5.66 & 3.46 & 9.77 & 40.07 \\
\hline & Water & 0 & 0.48 & 0 & 0 & 0.03 \\
\hline & Total pixel count & 3838 & 119436 & 836198 & 1195889 & 44731 \\
\hline
\end{tabular}

\begin{tabular}{|c|c|c|c|c|c|c|}
\hline & \multicolumn{5}{|c|}{ Manually identified pixels } \\
\hline & & Trees \& Shrubs & Arroyo & Water & Total pixel count & Percentage of Total \\
\hline \multirow{9}{*}{ 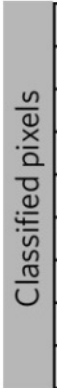 } & Villages before & 5.94 & 4.59 & 0.09 & 100085 & $3.89 \%$ \\
\hline & Villages after & 8.93 & 7.88 & 0.08 & 884457 & $34.38 \%$ \\
\hline & Agriculture & 6.45 & 8.89 & 0.02 & 784171 & $30.48 \%$ \\
\hline & Grassland & 8 & 0.73 & 0 & 199582 & $7.76 \%$ \\
\hline & Rocks & 2.53 & 1.21 & 0.02 & 95325 & $3.71 \%$ \\
\hline & Trees \& Shrubs & 7.05 & 2.97 & 0.04 & 73748 & $2.87 \%$ \\
\hline & Arroyo & 60.56 & 71.68 & 3.39 & 398508 & $15.49 \%$ \\
\hline & Water & 0.54 & 2.06 & 96.35 & 36996 & $1.44 \%$ \\
\hline & Total pixel count & 154894 & 184939 & 32947 & 2572872 & $100.00 \%$ \\
\hline
\end{tabular}

\section{Change Detection Results}

The Change Detection method delivered inconclusive results. The IKONOS imagery had changes shown for all change thresholds. Calculations of the number of pixels that increased were performed for each change percentage. At the $10 \%$ or greater threshold of change, the IKONOS NRB data had an increase in $81.86 \%$ pixels (Table 6) and a decrease of 6.47\% pixels (Figure 8). In Figure 8, pixels that increased in value (right image) are shown in green; decreases are shown in red. The NRG data had $10.17 \%$ of pixels increase (Table 7), while $16.88 \%$ of pixels decreased. At a $25 \%$ change threshold, $0.95 \%$ of pixels in the N/R/G data rose with a decrease of $4.91 \%$, while $20.11 \%$ of pixels increased and $5.15 \%$ decreased in the N/R/B data (Figure 9 ). In Figure 9 , villages are circled in the right image in white. There was no significant change in the villages. Visual analysis of the Change Detection data led to inconclusive results in regards to villages and burnt areas. Polygon outlines were layered over the results, but in all cases there was no clear pattern to discern by eye.

Landsat ETM+ imagery also had inconclusive results from the Change Detection. The Landsat Normalized Burn Ratio (BuRa) imagery had overwhelming increases in change at $5 \%$, and the thresholds for change were increased by $5 \%$ up to $50 \%$ to see if any patterns would emerge. At a $20 \%$ change or greater, the Landsat 432 data had $0.97 \%$ or pixels increase, and $1.55 \%$ decrease (Table 8 ). At the $35 \%$ threshold, change was negligible, with an increase of $0.03 \%$ of pixels and decrease of $0.47 \%$ of pixels. The BuRa data at the $20 \%$ threshold had a $75.29 \%$ of pixels increase, with $4.85 \%$ of pixels decreasing (Table 9 ).

At the $35 \%$ or greater threshold, the BuRa data had $24.90 \%$ of pixels increase, with $3.03 \%$ of pixels decreasing. Visual analysis proved to be inconclusive, similar to the IKONOS imagery. When examining the village outlines layered over the result data, some villages clearly had an increase in the BuRa, while other similarly sized and shaped villages had no change. The inconclusive results may have occurred due to the fluctuation of rainy and dry seasons. In 2000 the Darfur region experienced a drought, which was confirmed using TRMM data analysis, and between 2000 and 2007 rainfall remained at relatively low levels, with the rainy season shrinking from four months (May-September) down to July and August only. ${ }^{37}$ TRMM is the Tropical Rainfall Measuring Mission, a satellite used to monitor and collect rainfall data. In addition, the Landsat ETM+ imagery was not collected at the same time period. In 2000 imagery was collected on October 17, up to possibly 


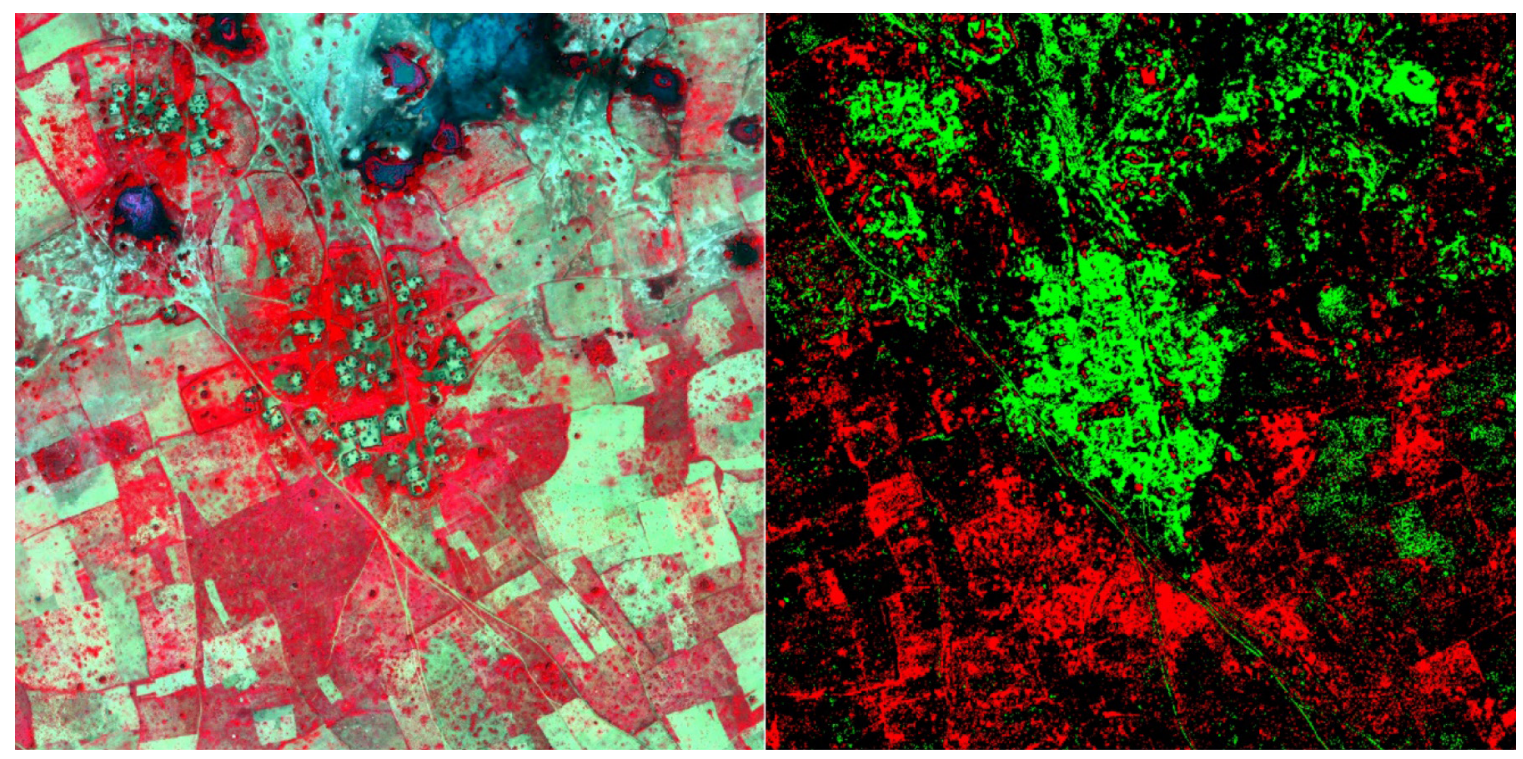

Figure 8. Change Detection results showing a 10\% threshold.

Satellite imagery courtesy of the DigitalGlobe Foundation / GeoEye.

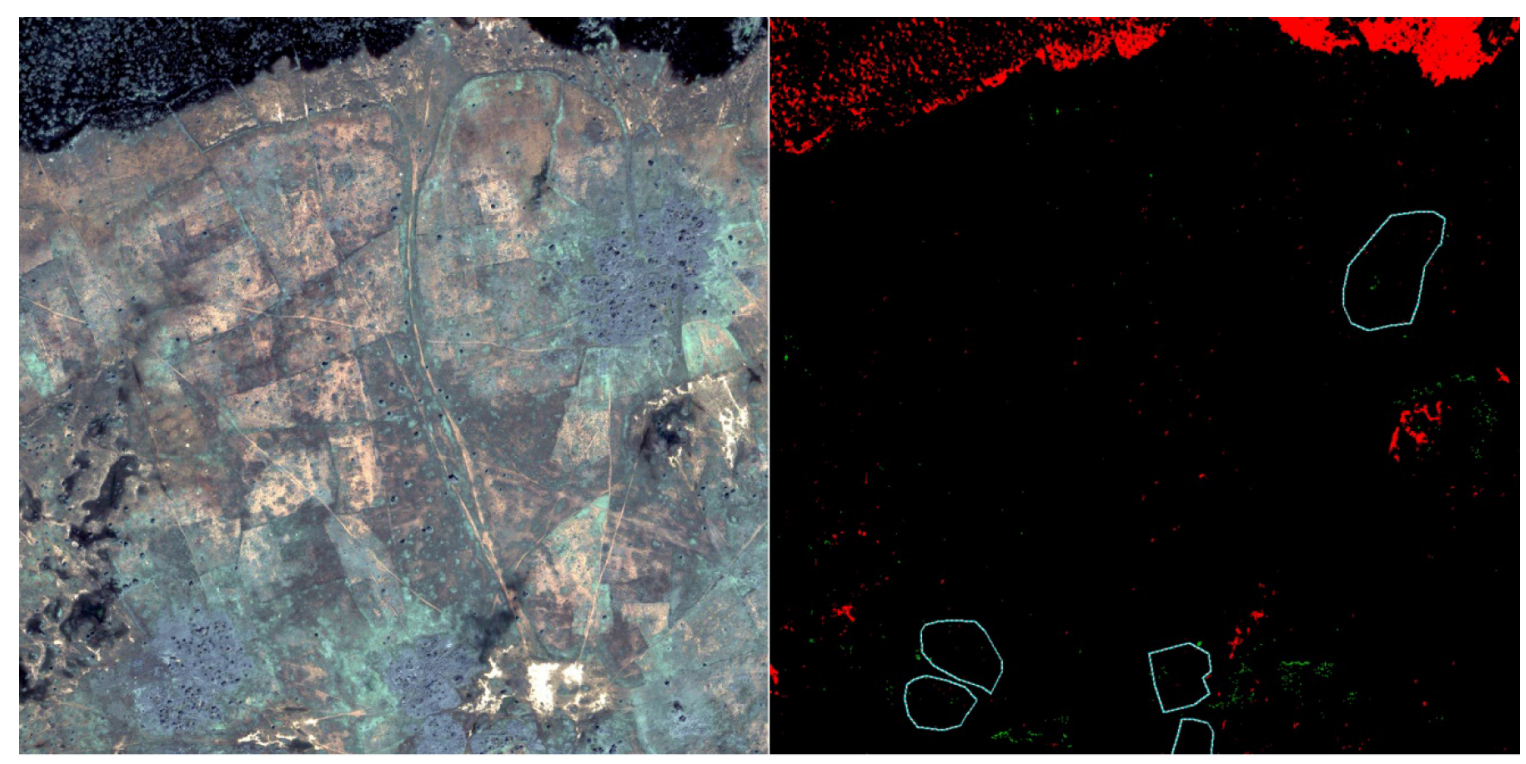

Figure 9. Change Detection results showing a 25\% threshold.

Satellite imagery courtesy of the DigitalGlobe Foundation / GeoEye. 
Table 6. Change Detection results for IKONOS NRB (Near Infrared, Red, Blue) imagery.

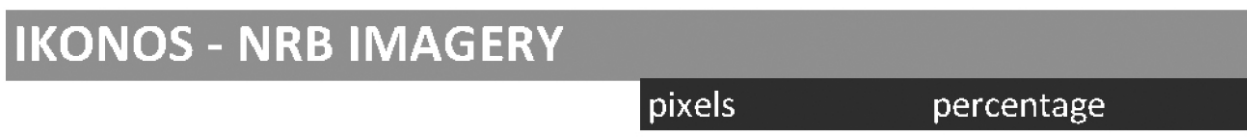

\begin{tabular}{|c|c|c|c|}
\hline \multirow[t]{3}{*}{$5 \%$} & increase & 188761931 & $89.48 \%$ \\
\hline & decrease & 14689650 & $6.96 \%$ \\
\hline & total & 210962241 & \\
\hline \multirow[t]{3}{*}{$10 \%$} & increase & 172694206 & $81.86 \%$ \\
\hline & decrease & 13636913 & $6.46 \%$ \\
\hline & total & 210962241 & \\
\hline \multirow[t]{3}{*}{$15 \%$} & increase & 138195692 & $65.51 \%$ \\
\hline & decrease & 12671106 & $6.01 \%$ \\
\hline & total & 210962241 & \\
\hline \multirow[t]{3}{*}{$20 \%$} & increase & 87748643 & $41.59 \%$ \\
\hline & decrease & 11747926 & $5.57 \%$ \\
\hline & total & 210962241 & \\
\hline \multirow[t]{3}{*}{$25 \%$} & increase & 42418173 & $20.11 \%$ \\
\hline & decrease & 10860276 & $5.15 \%$ \\
\hline & total & 210962241 & \\
\hline \multirow[t]{3}{*}{$30 \%$} & increase & 17784252 & $8.43 \%$ \\
\hline & decrease & 10011995 & $4.75 \%$ \\
\hline & total & 210962241 & \\
\hline \multirow[t]{3}{*}{$35 \%$} & increase & 7865557 & $3.73 \%$ \\
\hline & decrease & 9182877 & $4.35 \%$ \\
\hline & total & 210962241 & \\
\hline \multirow[t]{3}{*}{$40 \%$} & increase & 3344800 & $1.59 \%$ \\
\hline & decrease & 8360078 & $3.96 \%$ \\
\hline & total & 210962241 & \\
\hline \multirow[t]{3}{*}{$45 \%$} & increase & 1391394 & $0.66 \%$ \\
\hline & decrease & 7530360 & $3.57 \%$ \\
\hline & total & 210962241 & \\
\hline \multirow[t]{3}{*}{$50 \%$} & increase & 515260 & $0.24 \%$ \\
\hline & decrease & 6694053 & $3.17 \%$ \\
\hline & total & 210962241 & \\
\hline
\end{tabular}

This table shows the results of Change Detection by threshold percentage, listed on the left. The pixels column shows the number of pixels in the image that increased or decreased in spectral value at or higher than the threshold percentage. The percentage column shows the percentage of total pixels for the image that changed spectral value for each change threshold. 
Table 7. Change Detection results for IKONOS NRG (Near Infrared, Red, Green) imagery.

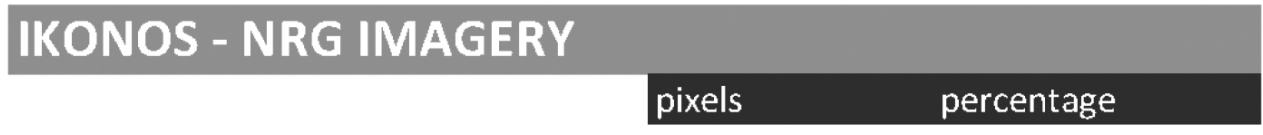

\begin{tabular}{|c|c|c|c|}
\hline $5 \%$ & increase & 50632869 & $24.00 \%$ \\
\hline & decrease & 66716271 & $31.62 \%$ \\
\hline & total & 210962241 & \\
\hline \multirow[t]{3}{*}{$10 \%$} & increase & 21464315 & $10.17 \%$ \\
\hline & decrease & 35609772 & $16.88 \%$ \\
\hline & total & 210962241 & \\
\hline \multirow[t]{3}{*}{$15 \%$} & increase & 8422701 & $3.99 \%$ \\
\hline & decrease & 20398548 & $9.67 \%$ \\
\hline & total & 210962241 & \\
\hline \multirow[t]{3}{*}{$20 \%$} & increase & 3614651 & $1.71 \%$ \\
\hline & decrease & 13792281 & $6.54 \%$ \\
\hline & total & 210962241 & \\
\hline \multirow[t]{3}{*}{$25 \%$} & increase & 2018248 & $0.96 \%$ \\
\hline & decrease & 10357915 & $4.91 \%$ \\
\hline & total & 210962241 & \\
\hline \multirow[t]{3}{*}{$30 \%$} & increase & 1498172 & $0.71 \%$ \\
\hline & decrease & 8003561 & $3.79 \%$ \\
\hline & total & 210962241 & \\
\hline \multirow[t]{3}{*}{$35 \%$} & increase & 1282507 & $0.61 \%$ \\
\hline & decrease & 6418611 & $3.04 \%$ \\
\hline & total & 210962241 & \\
\hline \multirow[t]{3}{*}{$40 \%$} & increase & 1052952 & $0.50 \%$ \\
\hline & decrease & 5475760 & $2.60 \%$ \\
\hline & total & 210962241 & \\
\hline \multirow[t]{3}{*}{$45 \%$} & increase & 718539 & $0.34 \%$ \\
\hline & decrease & 4756362 & $2.25 \%$ \\
\hline & total & 210962241 & \\
\hline \multirow[t]{3}{*}{$50 \%$} & increase & 378217 & $0.18 \%$ \\
\hline & decrease & 3578885 & $1.70 \%$ \\
\hline & total & 210962241 & \\
\hline
\end{tabular}

This table shows the results of Change Detection by threshold percentage, listed on the left. The pixels column shows the number of pixels in the image that increased or decreased in spectral value at or higher than the threshold percentage. The percentage column shows the percentage of total pixels for the image that changed spectral value for each change threshold. 
Table 8. Change Detection results for Landsat 432 imagery.

\section{LANDSAT - 432 IMAGERY}

pixels

percentage

\begin{tabular}{|c|c|c|c|}
\hline $5 \%$ & increase & 33182041 & $14.31 \%$ \\
\hline & decrease & 55669862 & $24.00 \%$ \\
\hline & total & 231937721 & \\
\hline \multirow[t]{3}{*}{$10 \%$} & increase & 14071238 & $6.07 \%$ \\
\hline & decrease & 33062395 & $14.25 \%$ \\
\hline & total & 231937721 & \\
\hline \multirow[t]{3}{*}{$15 \%$} & increase & 9428343 & $4.07 \%$ \\
\hline & decrease & 20308051 & $8.76 \%$ \\
\hline & total & 231937721 & \\
\hline \multirow[t]{3}{*}{$20 \%$} & increase & 7359853 & $3.17 \%$ \\
\hline & decrease & 11767553 & $5.07 \%$ \\
\hline & total & 231937721 & \\
\hline \multirow[t]{3}{*}{$25 \%$} & increase & 3958626 & $1.71 \%$ \\
\hline & decrease & 7677445 & $3.31 \%$ \\
\hline & total & 231937721 & \\
\hline \multirow[t]{3}{*}{$30 \%$} & increase & 1119488 & $0.48 \%$ \\
\hline & decrease & 5226545 & $2.25 \%$ \\
\hline & total & 231937721 & \\
\hline \multirow[t]{3}{*}{$35 \%$} & increase & 227627 & $0.10 \%$ \\
\hline & decrease & 3941835 & $1.70 \%$ \\
\hline & total & 231937721 & \\
\hline \multirow[t]{3}{*}{$40 \%$} & increase & 68499 & $0.03 \%$ \\
\hline & decrease & 2635863 & $1.14 \%$ \\
\hline & total & 231937721 & \\
\hline \multirow[t]{3}{*}{$45 \%$} & increase & 47841 & $0.02 \%$ \\
\hline & decrease & 1641322 & $0.71 \%$ \\
\hline & total & 231937721 & \\
\hline \multirow[t]{3}{*}{$50 \%$} & increase & 39652 & $0.02 \%$ \\
\hline & decrease & 842727 & $0.36 \%$ \\
\hline & total & 231937721 & \\
\hline
\end{tabular}

This table shows the results of Change Detection by threshold percentage, listed on the left. The pixels column shows the number of pixels in the image that increased or decreased in spectral value at or higher than the threshold percentage. The percentage column shows the percentage of total pixels for the image that changed spectral value for each change threshold. 
Table 9. Change Detection results for Landsat Normalized Burn Ratio imagery.

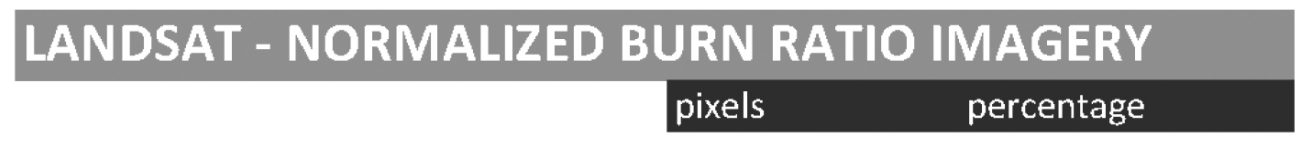

\begin{tabular}{|c|c|c|c|}
\hline $5 \%$ & increase & 36676605 & $63.24 \%$ \\
\hline & decrease & 1981967 & $3.42 \%$ \\
\hline & total & 57992061 & \\
\hline \multirow[t]{3}{*}{$10 \%$} & increase & 35680123 & $61.53 \%$ \\
\hline & decrease & 1933714 & $3.33 \%$ \\
\hline & total & 57992061 & \\
\hline \multirow[t]{3}{*}{$15 \%$} & increase & 33551679 & $57.86 \%$ \\
\hline & decrease & 1921691 & $3.31 \%$ \\
\hline & total & 57992061 & \\
\hline \multirow[t]{3}{*}{$20 \%$} & increase & 29514444 & $50.89 \%$ \\
\hline & decrease & 1902601 & $3.28 \%$ \\
\hline & total & 57992061 & \\
\hline \multirow[t]{3}{*}{$25 \%$} & increase & 23092557 & $39.82 \%$ \\
\hline & decrease & 1729253 & $2.98 \%$ \\
\hline & total & 57992061 & \\
\hline \multirow[t]{3}{*}{$30 \%$} & increase & 15781727 & $27.21 \%$ \\
\hline & decrease & 1509018 & $2.60 \%$ \\
\hline & total & 57992061 & \\
\hline \multirow[t]{3}{*}{$35 \%$} & increase & 9782117 & $16.87 \%$ \\
\hline & decrease & 1188746 & $2.05 \%$ \\
\hline & total & 57992061 & \\
\hline \multirow[t]{3}{*}{$40 \%$} & increase & 5614822 & $9.68 \%$ \\
\hline & decrease & 741897 & $1.28 \%$ \\
\hline & total & 57992061 & \\
\hline \multirow[t]{3}{*}{$45 \%$} & increase & 2928586 & $5.05 \%$ \\
\hline & decrease & 332728 & $0.57 \%$ \\
\hline & total & 57992061 & \\
\hline \multirow[t]{3}{*}{$50 \%$} & increase & 1281840 & $2.21 \%$ \\
\hline & decrease & 126961 & $0.22 \%$ \\
\hline & total & 57992061 & \\
\hline
\end{tabular}

This table shows the results of Change Detection by threshold percentage, listed on the left. The pixels column shows the number of pixels in the image that increased or decreased in spectral value at or higher than the threshold percentage. The percentage column shows the percentage of total pixels for the image that changed spectral value for each change threshold. 
three months after the rainy season had ended. In 2006 imagery was collected on August 31, which was at the tail end of the rainy season. Accurate data was not found for rainfall amounts for those two years, but because of the drought it is possible that Change Detection did not produce conclusive results because of the change in vegetation and moisture content in the area.

\section{Feature Extraction Results}

The N/R/G Feature Extraction resulted in a total of 2,676 correctly identified huts (out of 3,190 handcounted huts), for a success rate of $83.89 \%$ (Figure 10). The Feature Extraction also incorrectly identified 1,384 polygons/points as huts outside of villages. This results in $34.09 \%$ features identified by the Feature Extraction as false-positive. In Figure 10, huts identified by feature extraction (red dots) are compared to manually identified huts (green dots) in a village in the study area. Features were extracted using the Feature Analyst module in ERDAS IMAGINE.

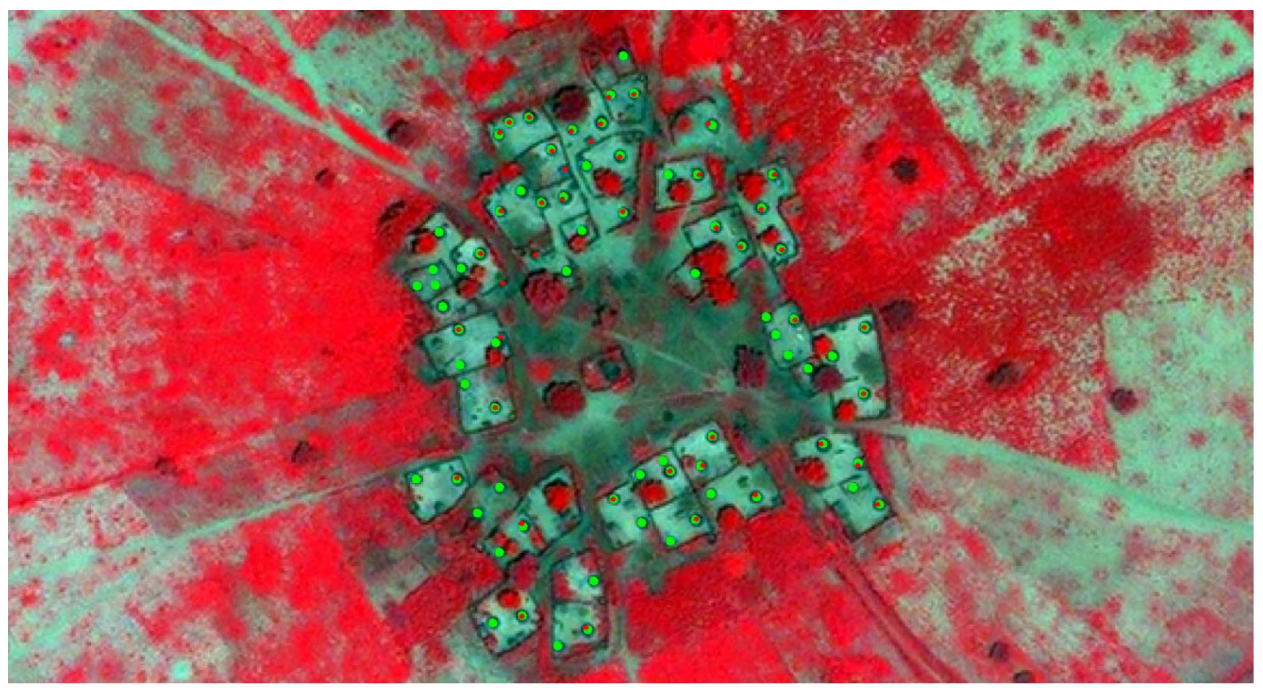

Figure 10. Feature Extraction results in a sample village., IKONOS imagery dated September 16, 2004.

Satellite imagery courtesy of the DigitalGlobe Foundation / GeoEye.

The N/R/B Feature Extraction resulted in a total of 1,513 correctly identified huts (out of 3,190 handcounted huts), for a success rate of $47.43 \%$. The Feature Extraction also incorrectly identified 326 polygons/ points as huts outside of the villages, resulting in a $17.73 \%$ false-positive rate.

The features brought into ArcMap were analyzed using a kernel density module, resulting in a visual graphic showing where the features are concentrated (Figure 11).

This image was layered over the true-color pan-sharpened image, and the transparency reduced to $50 \%$, allowing for the geography of the area to show how it correlates to the kernel density image. A visual analysis of the images shows that the clusters of huts can be identified by the kernel density image - false positives occur where incorrectly identified features are located, particularly in the South East corner of the study area where the arroyo is, however the false positives are not as clustered as the huts and do not affect the kernel density image as the geometry of the shapes indicate natural features (Figure 12).

A count of villages combined with a count of hotspots yields two results. Clouds in the southwest corner of the study area prevented Feature Extractions from occurring in that area, as no points were selected during the process. Seventy seven villages were counted in the study area, and 43 hot spots were counted after the kernel density function was performed on the NRB imagery, resulting in an accuracy of $55.8 \%$ of hotspots matching villages. However, if the villages in the cloud region are taken out, 54 villages are counted, and the resulting accuracy of hotspots matching villages is $79.6 \%$. As clouds were not masked in this study, any villages under cloud cover would have been segmented from the village count if the masking had been performed.

Feature Extraction, when combined with the kernel density "hot spot" method, appears to be a useful tool to identify villages. Unfortunately for this study (and for the Darfuri affected), the 2006 high resolution imagery had no surviving villages or huts in the study area. Because of this, Feature Extraction could not be 


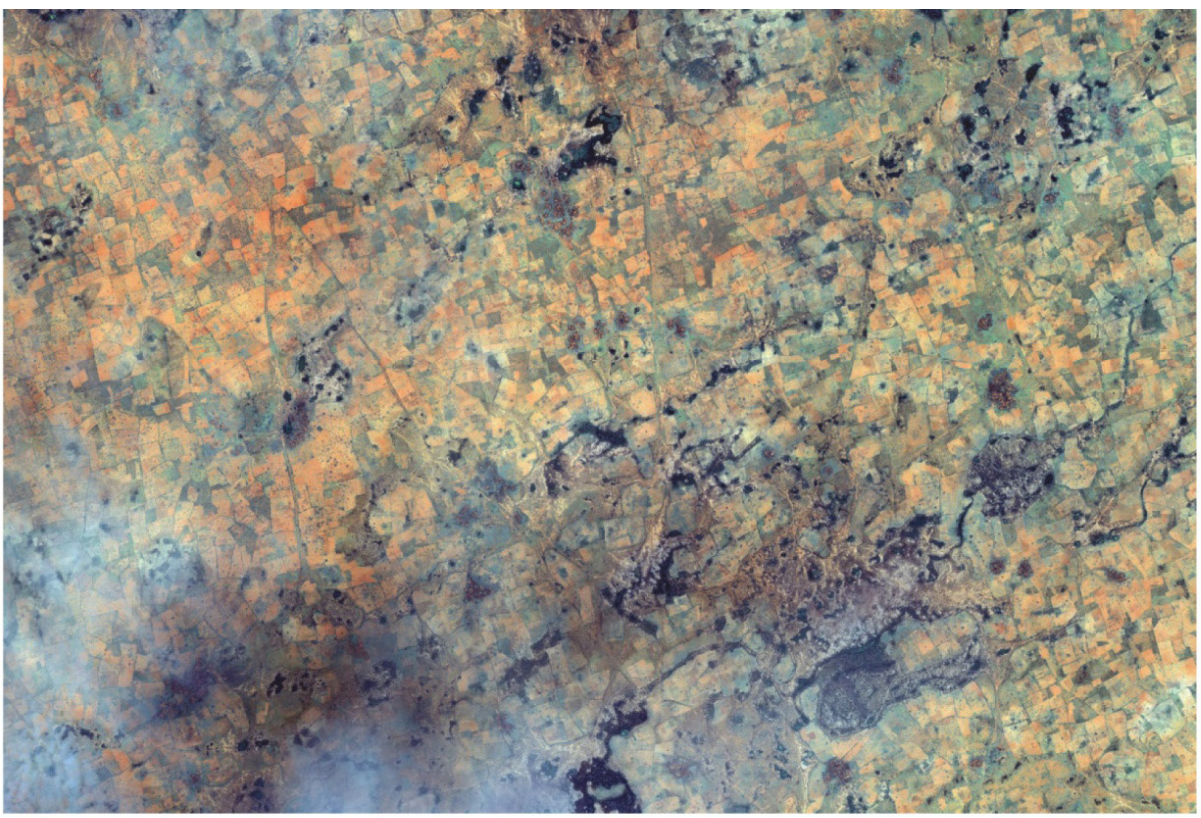

Base layer showing satellite imagery, above.

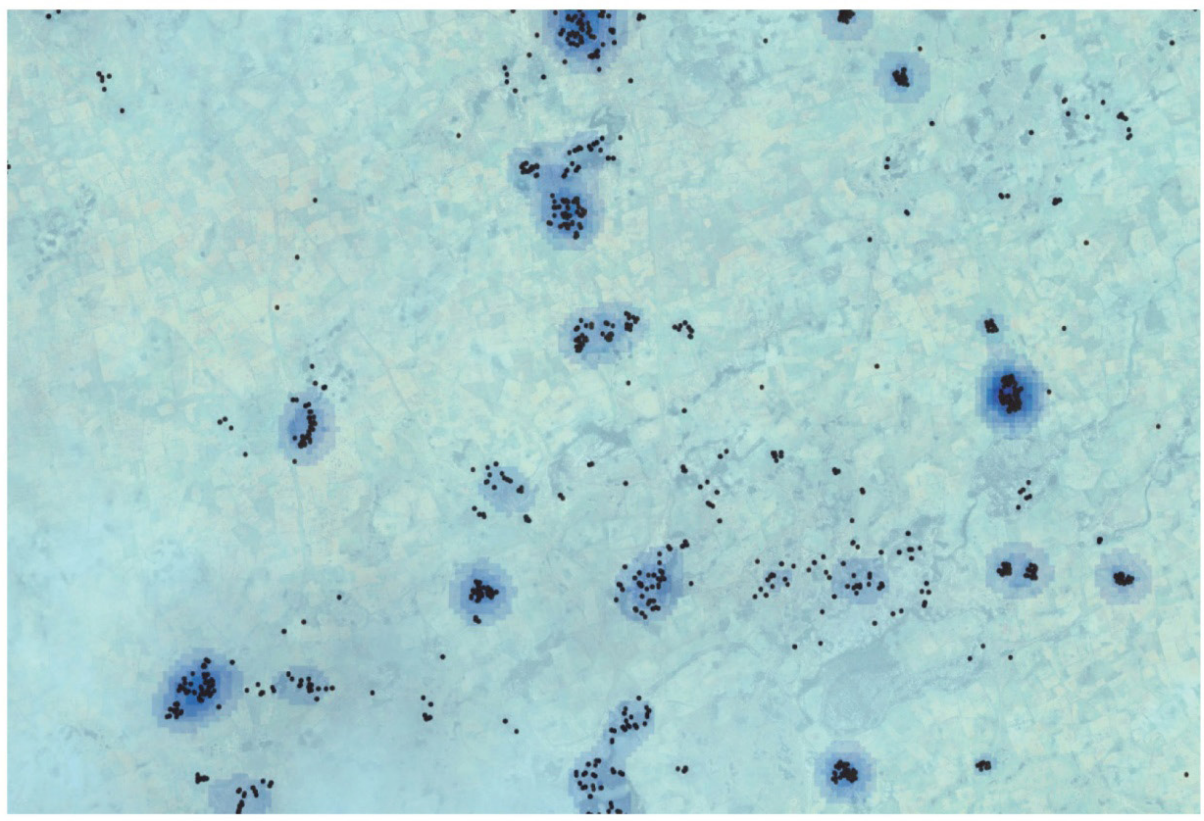

Kernel Density Estimation 'hotspots' over satellite imagery with extracted features (black dots), above.

Figure 11. Kernel Density Estimation overlaying satellite imagery. Satellite imagery courtesy of the DigitalGlobe Foundation / GeoEye.

performed on the after imagery, and an accurate assessment of the extraction/kernel density method cannot be ascertained. The results also indicate that clouds should be masked before the Feature Extraction and village counting is performed, even though some villages are visible in the cloud covered area. However, the visual analysis of the kernel density results points to its usefulness when identifying villages, and in theory, villages that no longer exist in the after imagery would not have the clustering of features that the before imagery would depict. A custom Feature Extraction algorithm could also have a positive effect on the accuracy of identifying huts, as the Feature Extraction tools used in this study were part of the standard Feature Analyst toolset available for ERDAS IMAGINE. 


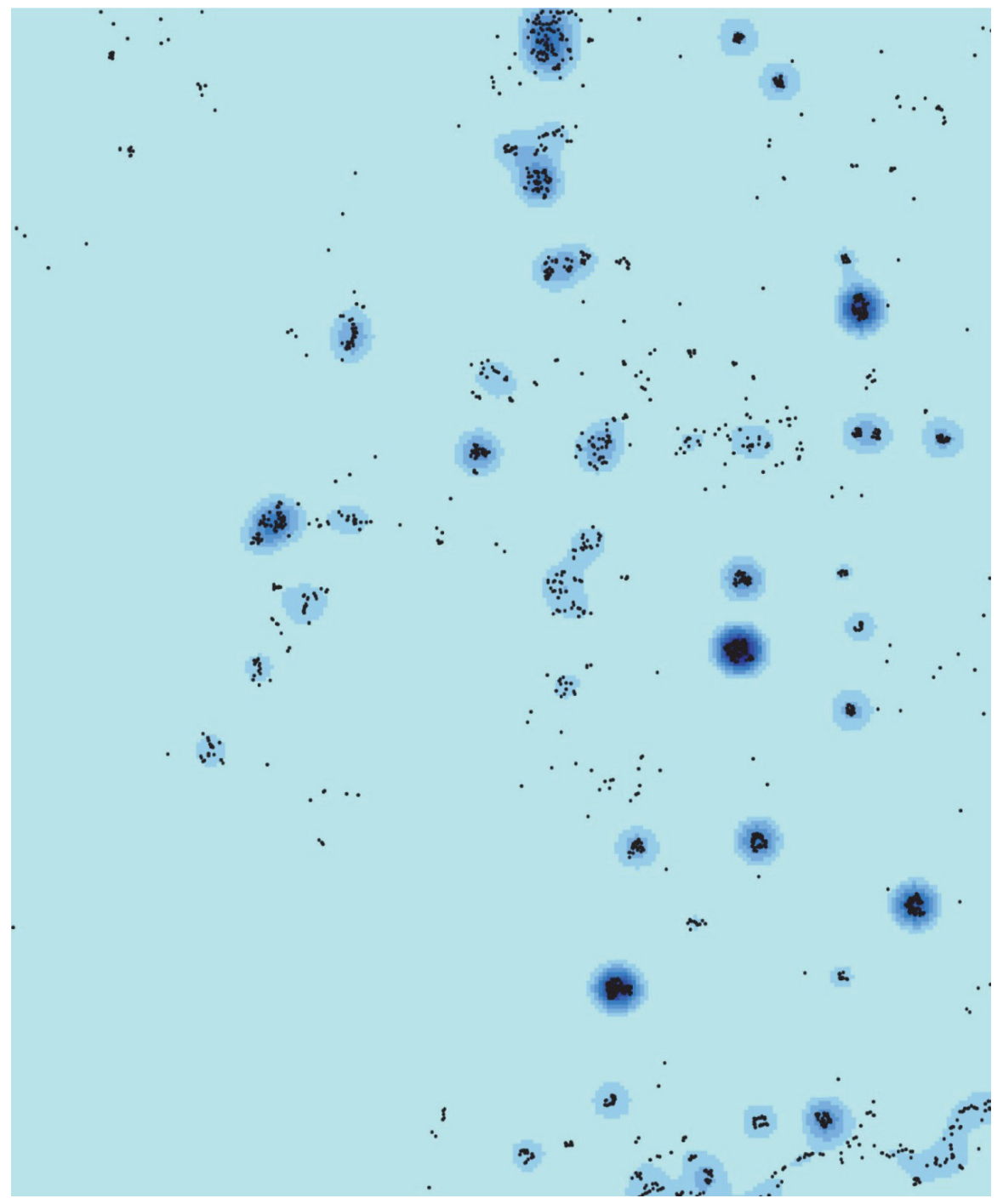

Figure 12. Village Hotspot map through kernel density.

\section{Conclusions}

Satellite imagery and remote sensing methods offer much potential in verifying and tracking human rights violations and mass violence. The results of this study indicate that Feature Extraction is a useful ancillary tool to the practice of manual identification, particularly in places similar to Darfur with semi-arid grassland and rural huts and villages (Table 1). In addition, as this study used a stock algorithm in the Feature Analyst software module, custom algorithms could be produced that offer much higher accuracy rates. While the data difficulties in this study resulted in inconclusive results, the outcome suggests that both before and after imagery of burned villages can be examined using a combination of Feature Extraction and the kernel density function to identify villages. The before and after kernel density maps theoretically would be compared for change, and any missing "hotspots" could indicate a burned village and warrant further examination. In addition, the results of the Feature Extraction method indicate that masking of clouds, normally a common practice, gives more positive results than non-masking.

While some research suggests that geospatial analysis in the field of human rights has slowly increased in the past decade, other research indicates that these methods are being performed by a growing number of organizations on a variety of cases. This growth is exciting for the human rights and remote sensing fields, as these studies continue to be important due to human rights violations across the world. The use of satellite imagery is one tool (out of many) that human rights workers can use for corroboration of mass violence, as well as evidence of past mass violence in criminal trials. As of the writing of this article, Darfur is still 
experiencing violence between the government, rebels, and various tribes. The $\mathrm{BBC}$ reports that as of May 2013 approximately 300,000 people have died since the most recent wave of violence started in 2003, and over 1.4 million people are homeless, either as foreign refugees or IDPs. ${ }^{38}$ The International Criminal Court issued an arrest warrant for President al-Bashir in March of 2009, ${ }^{39}$ on charges of war crimes and crimes against humanity based on his role in the Darfur crisis. However because of the intricacies of international law, the arrest warrant was delivered to the Sudanese government, who most likely will not execute the warrant. ${ }^{40}$ While diplomacy and international politics play a major role in stopping and holding governments responsible for human rights violations, remote sensing also plays an important role in verification. Combined with human rights organization information from groups like Amnesty International or the United Nations Human Rights Council, satellite imagery and remote sensing methods can provide the world a window into previously unseen areas where atrocities occur.

\section{Endnotes}

1 Walter Laquer, The Terrible Secret: Suppression of the Truth About Hitler's "Final Solution," (New York: Henry Holt and Company, Inc, 1988), 6-14.

2 Jamie F. Metzl, “Information Technology and Human Rights," Human Rights Quarterly 18:4 (1996): 705-46.

3 Metzl, "Information Technology," 707-08.

4 Matthew Levinger, "Geographical Information Systems Technology as a Tool for Genocide Prevention: The Case of Darfur," Space and Polity 13:1 (2009): 69-76.

5 Prins Engeneering, "Use of low cost Landsat ETM+ to Spot Burnt villages in Darfur, Sudan." International Journal of Remote Sensing 24:4 (2008): 1207-14.

6 Humanitarian Information Unit, "Sudan (Darfur) - Chad Border Region Confirmed Damaged and Destroyed Villages: U.S. Department of State, Humanitarian Information Unit (November 15, 2004)." Accessed April 2, 2013. https://hiu.state.gov/ Products/ChadSudan_DamagedDestroyedVillages_2004Nov15_HIU.pdf.

7 Andrew Marx and Samuel Goward, "Remote Sensing in Human Rights and International Humanitarian Law Monitoring: Concepts and Methods," Geographical Review 103:1 (2013): 108-10.

8 Florent Mouillot, Martin G. Schultz, Chao Yue, Patricia Cadule, Kevin Tansey, Philippe Ciais, and Emilio Chuvieco, "Ten Years of Global Burned Area Products From Spaceborne Remote Sensing - A Review: Analysis of User Needs and Recommendations for Future Developments," International Journal of Applied Earth Observation and Geoinformation 26 (2014): 67.

9 John J. Sulik and Scott Edwards, "Feature Extraction for Darfur: Geospatial Applications in the Documentation of Human Rights Abuses." International Journal of Remote Sensing 31:10 (2010): 2521-33.

10 Sulik and Edwards, "Feature extraction for Darfur," 2522-3.

11 Sulik and Edwards, "Feature extraction for Darfur," 2531.

12 For in depth historical analyses on the crisis in Darfur, see Mahmood Mamdani, Saviors and Survivors: Darfur, Politics, and The War On Terror (New York: Doubleday, 2009); John Hagan and Wenona Rymond-Richmond, Darfur and the Crime of Genocide (Cambridge: Cambridge University Press, 2009); Gerard Prunier, Darfur: A 21st Century Genocide (Ithaca, NY: Cornell University Press, 2008).

13 Amnesty International, “Eyes on Darfur: Donkey Dereis.” Accessed April 7, 2012. http://www.eyesondarfur.org/satellite.html.

14 Joshua Project, "People-in-Country Profile: Masalit of Sudan." Accessed April 7, 2012. (WherLink)(http://www.joshuaproject. net/people-profile.php?peo3=13578\&rog3=SU).

15 Amnesty International, "Eyes on Darfur."

16 Radio Dabanga, “UNAMID: Abdel Wahid Gunmen Threaten Kalma Camp Leaders Over Doha Participation,” Radio Dabanga, July 26, 2010. Accessed May 23, 2013. http://www.radiodabanga.org/node/2509.

17 Amnesty International, "Eyes on Darfur."

18 AAAS, "AAAS Scientific Responsibility, Human Rights and Law Program; Geospatial Technologies and Human Rights; Darfur, Sudan and Chad: Conflict Monitoring Case Study Summary (2007)," Accessed March 5, 2013. http://shr.aaas.org/ geotech/darfur/darfur_appendix.shtml.

19 Signal Program on Human Security and Technology, "Sudan: Anatomy of a Conflict." Accessed September 4, 2014. http://hhi. harvard.edu/sites/default/files/publications/Sudan\%20Anatomy\%20of\%20a\%20Conflict Signal\%20(1).pdf.

20 The Guardian, “They escaped the Janjaweed. Now Darfur's returning villagers face real fight to survive," The Guardian, September 7, 2004. Accessed September 10, 2014. http://www.theguardian.com/society/2004/sep/07/internationalaidanddevelopment.sudan.

21 The DigitalGlobe Foundation: http://www.digitalglobefoundation.org/.

22 DigitalGlobe, "IKONOS Datasheet and Specifications," Accessed March 5, 2013. http://www.geoeye.com/CorpSite/products/ imagery-sources/Default.aspx\#ikonos.

23 USGS (United States Geological Survey), "Landsat Missions; SLC-off Products: Background," Accessed May 22, 2013. http:// landsat.usgs.gov/products_slcoffbackground.php. 
24 Sulik and Edwards, "Feature extraction for Darfur," 2524-5.

25 C. H. Key and N. C. Benson. "Measuring and Remote Sensing of Burn Severity: the CBI and NBR,” Poster, 1999. Accessed May 13, 2013. http://www.nrmsc.usgs.gov/files/norock/products/SEVER36_im_copy6.pdf.

26 Ibid.

27 USGS, "Landsat Missions."

28 Ibid.

29 Sulik and Edwards, "Feature extraction for Darfur."

30 Thomas M. Lillesand, Ralph W. Kiefer, and Jonathan W. Chipman, Remote Sensing and Image Interpretation (Hoboken, NJ: John Wiley \& Sons, Inc, 2006), 554.

31 K. Perumal and R. Bhaskaran, "Supervised Classification: Performance of Multispectral Images," Journal of Computing 2:2 (2010): 124-129.

32 Lillesand et al., "Remote Sensing," 551.

33 Perumal and Bhaskaran, "Supervised Classification." 127.

34 Ibid.

35 Lillesand et al., "Remote Sensing." 595.

36 Sulik and Edwards, "Feature extraction for Darfur."

37 Yale University Genocide Studies Program: Remote Sensing Project, "Tracking the Genocide in Darfur: Population Displacement as Recorded by Remote Sensing," Working Paper, 2009. Accessed March 31, 2013. http://www.yale.edu/gsp/ gis-files/darfur/Tracking-Genocide-in-Darfur-by-Remote-Sensing_No.36.pdf.

38 BBC News, "Darfur conflict displaces 300,000 in five months - UN," BBC News, May 23, 2013. Accessed May 24, 2013. http:// www.bbc.co.uk/news/world-africa-22649076.

39 BBC News, "Warrant issued for Sudan's leader," BBC News, March 4, 2009. Accessed May 24, 2013. http://news.bbc.co.uk/2/ hi/africa/7923102.stm.

40 Ibid. 